\title{
High dimensional geometry of fitness landscapes identifies master regulators of evolution and the microbiome
}

Holger Eble, ${ }^{1}$ Michael Joswig, ${ }^{1,2 *}$ Lisa Lamberti ${ }^{3,4}$,William B. Ludington ${ }^{5,6 *}$

${ }^{1}$ Chair of Discrete Mathematics/Geometry, TU Berlin, Germany

${ }^{2}$ MPI MiS Leipzig, Germany

${ }^{3}$ Department of Biosystems Science and Engineering, ETH Zürich, Basel, Switzerland

${ }^{4}$ SIB Swiss Institute of Bioinformatics, Basel, Switzerland

${ }^{5}$ Department of Embryology, Carnegie Institution for Science, USA

${ }^{6}$ Department of Biology, Johns Hopkins University, Baltimore, MD, USA

*To whom correspondence should be addressed;

E-mail: joswig@math.tu-berlin.de, ludington@carnegiescience.edu.

\begin{abstract}
A longstanding goal of biology is to identify the key genes and species that critically impact evolution, ecology, and health. Yet biological interactions between genes $(1,2)$, species (3-6), and different environmental contexts (7-9) change the individual effects due to non-additive interactions, known as epistasis. In the fitness landscape concept, each gene/organism/environment is modeled as a separate biological dimension (10), yielding a high dimensional landscape, with epistasis adding local peaks and valleys to the landscape. Massive efforts have defined dense epistasis networks on a genome-wide scale (2), but these
\end{abstract}


have mostly been limited to pairwise, or two-dimensional, interactions (11). Here we develop a new mathematical formalism that allows us to quantify interactions at high dimensionality in genetics and the microbiome. We then generate and also reanalyze combinatorically complete datasets (two genetic, two microbiome). In higher dimensions, we find that key genes (e.g. pykF) and species (e.g. Lactobacillus plantarum) distort the fitness landscape, changing the interactions for many other genes/species. These distortions can fracture a "smooth" landscape with one optimal fitness peak into a landscape with many local optima, regulating evolutionary or ecological diversification (12), which may explain how a probiotic bacterium can stabilize the gut microbiome.

\section{Introduction}

A fitness landscape depicts biological fitness as a function of its many underlying parts, namely genes, each as a separate dimension $(10,13,14)$. Interactions between genes can change their individual impacts on fitness in a non-additive way (15), adding local peaks and valleys to the fitness landscape, which affects the evolutionary paths through the landscape (16). The mathematical frameworks to quantify biological interactions, namely epistasis, determine the degree of non-additivity, and the concept has been applied to genetics $(1,2)$, microbiomes $(3)$, and ecology (4-6). In two dimensions, epistasis calculates interactions between e.g. two genes as the degree to which a double mutant phenotype can be predicted by measuring the two single mutants independently. Applying epistasis to genome-wide measurement of pairwise $(17,18)$ and three-way (2) genetic interactions has revealed biochemical pathways composed of discrete sets of genes as well as complex traits, such as human height, that are affected by almost every gene in the genome $(19,20)$. New techniques allow epistasis to be applied to broader data types (21). 
Epistatic interactions can arise due to mutations $(13,14,22)$ or when sex, recombination, and horizontal gene transfer bring groups of genes together $(1,23-26)$, making multiple dimensions interact simultaneously. Interactions between bacteria in the microbiome also have functional consequences $(3,27-31)$ and are prevalent in higher-dimensions $(3,31)$, where community assembly may introduce groups of species in different combinations e.g. in a fecal transplant.

Interactions in higher dimensions could change the topography of the fitness landscape (31), and their relative importance is unknown. To various extents, current approaches are limited in their ability to discern the topography of interaction landscapes in high dimensions due to $(i)$ sign epistasis, which does not generalize well to more than two dimensions, (ii) a narrow ability to account for genomic context, and (iii) statistical considerations of the false discovery rate due to multiple testing $(32,33)$. Several different concepts of epistasis exist in the literature (34). However, standard epistasis frameworks often rely on parameter fitting, which brings along additional constraints (35). "Circuits", which can describe all possible epistatic interactions $(33,36)$, introduce false discovery rate challenges.

Here we develop a new formulation of the fitness cube concept $(10,13,14)$, where each biological entity (gene, organism, environmental factor) is a separate dimension (Fig. 1a). Because the biological entities are discrete (i.e., either a bacterium is there or it is not), our framework is discrete too. The cubes represent the landscape for interactions and can be composed of many dimensions as $n$-dimensional hypercubes. We then develop epistatic filtrations to locate the epistasis on this fitness landscape. Our approach solves problems of context and sign while reducing multiple testing concerns, all in a parameter-free form that is consistent across many dimensions (Fig. 1, Box 1). 
(a) 3D bi-allelic genotype

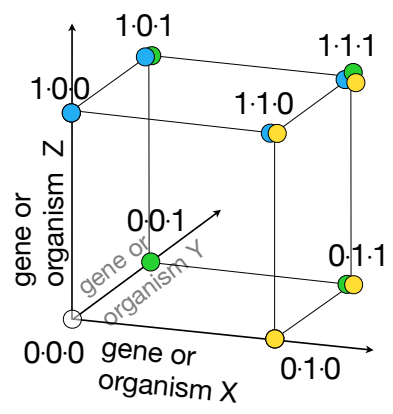

(c) Graph of simplices from (b) as nodes and bipyramids as edges

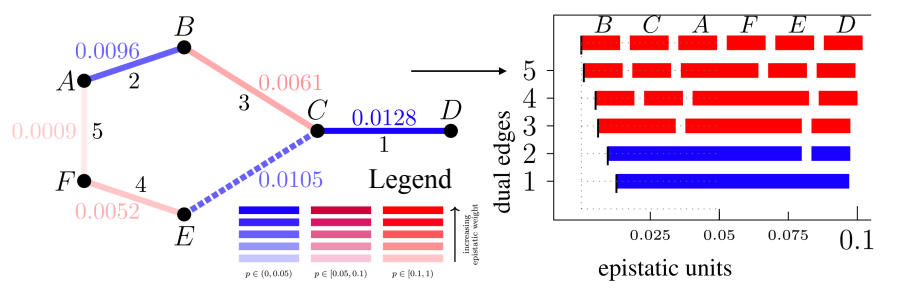

(b) Triangulation of $3 D$ genotype

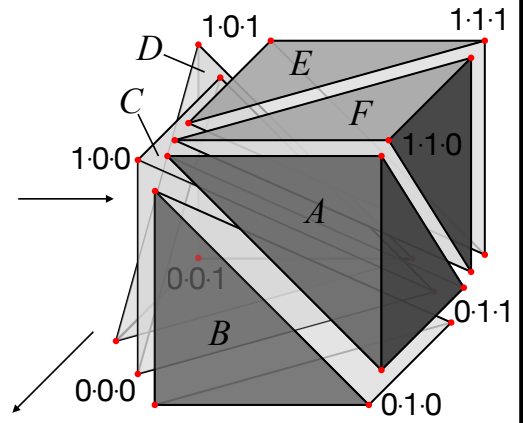

(d) Epistatic filtration of graph in (c) epistatic units
Box 1: a new definition of epistasis that is consistent in higher dimensions

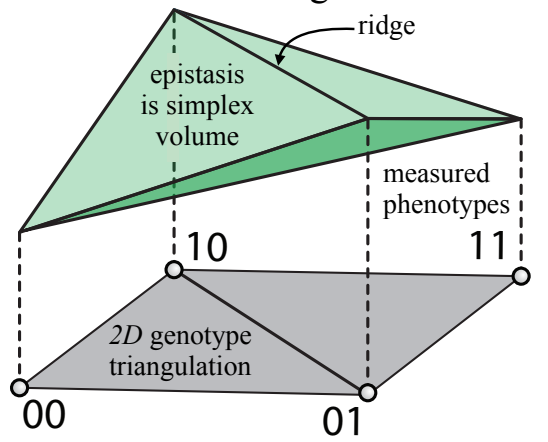

In a two gene, bi-allelic genetic landscape (gray), the fitness landscape is determined by the measured phenotypes for each genotype. These lifted points enclose a 3D simplex (green). The volume of the simplex is the epistasis between the two genes. The upper surface of this simplex forms a ridge, which induces the $2 \mathrm{D}$ triangulation, i.e. two gray triangles, which are the simplest shape into which the genetic landscape can be decomposed. The definition is consistent in higher dimensions. Note that the number of possible triangulations and resulting epistatic simplices is much larger in higher dimensions.

Figure 1: Filtrations describe epistatic topography. (a) Interacting biological entities, e.g. genes in a cell or bacterial strains in a microbiome, can be depicted as orthogonal dimensions in a unit cube, where vertices represent different genotypes or combinations of strains. (b) With 3 dimensions, the triangulation (14) of the fitness landscape produces $3 D$ simplices (labeled $A-F$ ) of the genotypes, and $4 D$ simplices of the fitness landscape (not shown) give the epistasis. (c) To map the global connectivity of the landscape, we merge adjacent simplices in a dual graph of the 3-cube triangulation, where nodes $A-F$ are the simplices from (d) and the edges are the volumes of the bipyramids from the merges of neighboring simplices. The smallest bipyramid, edge 5 , is formed first, followed by the next larger and so forth on up to the largest bipyramid, edge 1. The data set is from Esherichia coli mutations in topA, spoT, and pykF from (37). (c legend) Each dual edge has two parameters: its epistatic weight (indicated by shade) and its $p$-value (indicated by color). Black indices in (c) label the critical dual edges of $\mathcal{S}(h)$, where critical indicates that loss of the edge leaves nodes unconnected to the graph. (d) The sequence of merges between adjacent simplices (reading from top to bottom) shown in the dual graph is depicted by the epistatic filtration. Epistasis of the merged simplex is indicated by the thin, black vertical hatch mark on the far left bar of each row. Total width of the bars is fixed. Note the non-critical C+E merge is not depicted in the filtration because those simplices are already merged with $\mathrm{B}, \mathrm{A}$, and $\mathrm{F}$. 
60

61

We first decompose the fitness cube into its most elementary parts through a triangulation (Box 1, Fig. 1b). Triangulations are used e.g. in computer vision to decompose a surface, such as a human face, into discrete parts, which are triangles. Generalizing to higher dimensions, the triangles connecting genotypes are simplices (Fig. 1b). The volume of each simplex connotes the local steepness of the landscape (Box 1). To establish the global topography of the landscape we merge adjacent simplices in a stepwise manner such that flattest parts of the landscape are merged first and the steepest parts last (Fig. 1c). Each adjacent pair of merged simplices, $s$ and $t$, forms a bipyramid, $(s, t)$ through their shared face. The epistatic weight of $(s, t)$ is

$$
e_{h}(s, t):=\left|\operatorname{det} E_{h}(s, t)\right| \cdot \frac{\operatorname{nvol}(s \cap t)}{\operatorname{nvol}(s) \cdot \operatorname{nvol}(t)} .
$$

where $E_{h}(s, t)$ is the matrix specifying the vertices with their corresponding fitness phenotypes and nvol denotes the dimensionally normalized volume of the genotypes (Box 1; Appendix B1-B6). We use the notation

$$
\left\{v^{(1)}\right\}+\left\{v^{(2)}, \ldots, v^{(n+1)}\right\}+\left\{v^{(n+2)}\right\}
$$

for the bipyramid $(s, t)$, where the first and last vertices are the apices and the middle set forms the shared face. The $n+2$ genotypes of the bipyramid form a non-linear interaction of dimension $n$ when $e_{h}(s, t)>0$.

We visualize the topography of the epistatic landscape by forming a dual graph of $\mathcal{S}(h)$, where the nodes are the maximal simplices and adjacent simplices form the dual edges. Blue edges indicate epistasis (Fig. 1c). The epistatic filtration of $h$ (Fig. 1d) depicts the path from lowest to highest epistasis by merging adjacent simplices to form a connected cluster c.f. (38). In this sense, epistatic filtrations encode a global notion of epistasis in higher dimensions by 
connecting adjacent bipyramids. This method has many advantages over parameter fitting, including that it does not depend on the statistical constraints of determining a best fit.

Filtrations are also not constrained by the sign of epistasis, which depends on which genotype is considered wildtype, a somewhat arbitrary decision given varied ancestries (see Appendix B1). Studying adjacent simplices and their neighboring relationships, as we propose below, allows reconstruction of the fitness landscape and its epistatic properties in high dimensions. This process rests on the mathematical theory of linear optimization, convex polyhedra, and regular subdivisions (38).

We note that bipyramids account for the majority of genomic contexts (38), c.f. Table S1. Furthermore, the location(s) of inferred epistasis is robust to the choice of triangulation $\mathcal{S}(h)$ $(38)$.

\subsection{An evolutionary genetics example of epistatic filtrations}

To illustrate our approach, we examined an existing data set from Lenski's (39) classic experimental evolution of Esherichia coli, in a set of strains with each combination of five beneficial mutations (37) (Fig. 2a). We first examine $n=3$ loci, corresponding to biallelic mutations in topA, spoT, and pykF (Fig. 1c,d). Epistasis was generally low in magnitude (37,40), and occurs in two ways: $(i)$ either from merging groups of groups of simplices (c.f. BC + AFE in line \#2 of Fig. 1d), which indicates a complex interaction, or (ii) from merging a single simplex, c.f. D, with the aggregated rest of the simplices (c.f. line \#1 of Fig. 1d), much like a dominant effect in the NK model (14). This second way is consistent with a fitness landscape distortion, which occurs when certain mutations influence the interactions of many other genes (41). Geometrically, such a distortion constitutes a vertex split (42). We next add a fourth biallelic mutation, in the glmUS locus (Fig. 2b,c), encoding peptidoglycan availability, which is an essential component of the cell wall. 

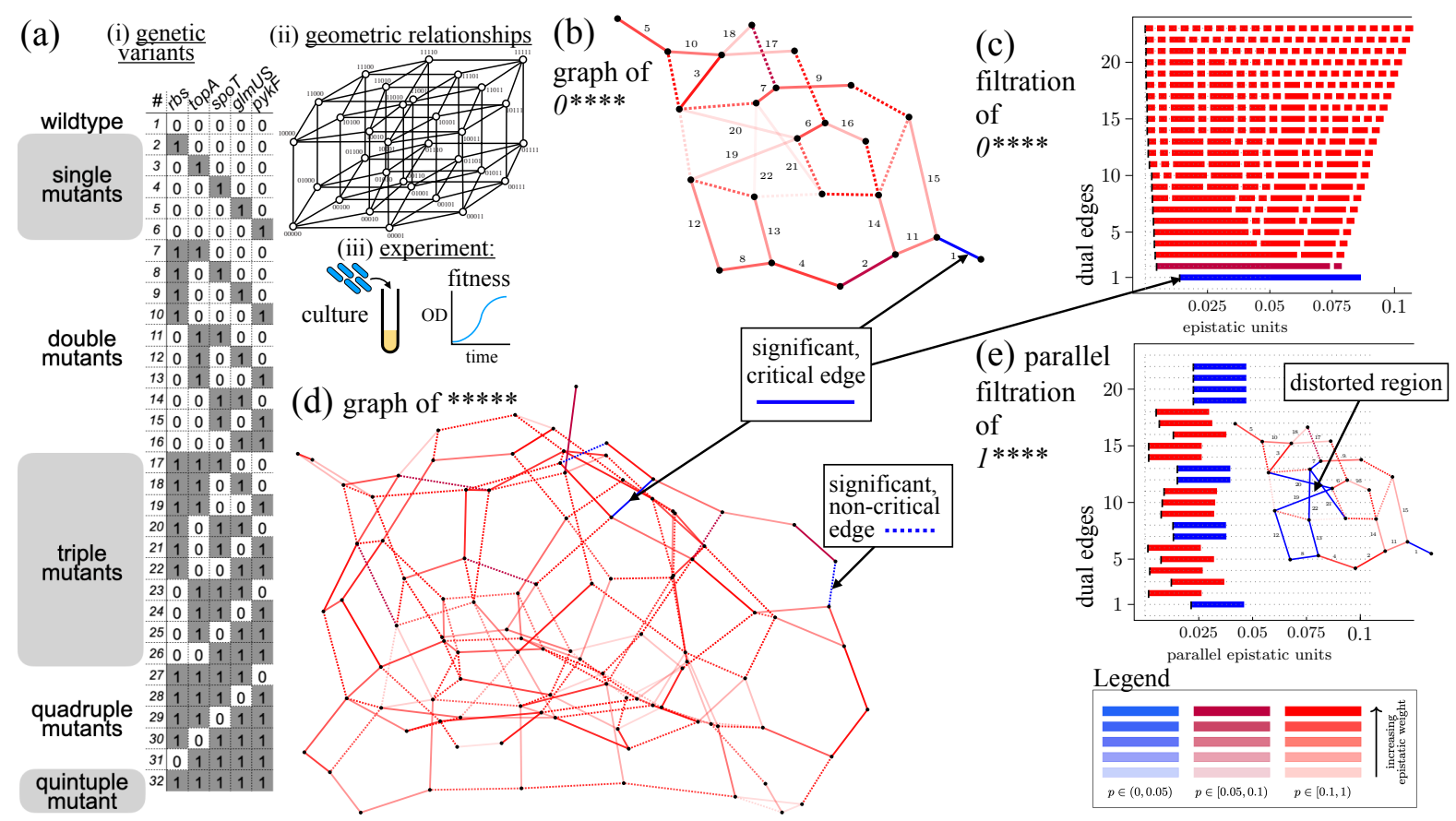

Figure 2: E. coli evolution is guided by epistatic landscape distortions. (a) (i) E. coli mutants examined (37), (ii) their geometric relationships, and (iii) experimental approach to measure fitness. (b) Edge labeled dual graph and (c) epistatic filtration restricted to $n=4$ mutations in topA (locus 2), spoT (locus 3), glmUS (locus 4) and pykF (locus 5). Locus 1, rbs, is fixed 0 (wildtype). Note that the left edge of the bars in (c) indicates there is very little epistatic weight added to the filtration except for the final merge, where the single genotype 00001 gives weight to the entire filtration. This final interaction corresponds to the vertices $\{00001\}+\{00000,01001,00101,00011\}+$ $\{00010\}$. (d) Dual graph for the complete Khan data set. Black indices in (b) label the critical dual edges of $\mathcal{S}(h)$. (e) In the parallel filtration, for $1 * * * *$, where the $r b s$ mutation is present, the landscape is disorted by a concentrated area of higher epistasis. Inset: graph in (b) recolored with weights from (e).

The filtration reveals a smooth, additive landscape with one dominant cell where epistasis arises only in the final merge of the filtration (Fig. 2c), meaning the epistatic topography of the entire landscape (Fig. 2d) rests upon the single vertex, 00001, pykF. While the previous analysis detected a significant, marginal effect of $p y k F$ (37), filtrations reveal the geometric structure in terms of which specific combinations of loci are responsible for the effect (Fig. 2e): the interaction between the $\operatorname{glm} U S,\{00001\}$, and $p y k F,\{00010\}$, genes requires the context of four loci, $\{00000,01001,00101,00011\}$, yet it involves only up to double mutants, suggesting high dimensional epistasis that arises from lower dimensional interactions (Fig. 2c). This conclusion 
is consistent with recent genome-wide work on trans-gene interactions (19), suggesting that complex traits may arise from genome-wide epistasis, where each mutation's contribution to the trait depends on the context of other mutations.

We introduced parallel transport $(38, \S 6.6)$ to give a geometric measure of context-dependence for the same set of loci with different bystanders (e.g. species or genes) (see Fig. S1), previously examined by conditional or marginal epistasis (43). Examining the Khan data with and without the pykF mutation (37) (Fig. S2) showed increased significance in 9 out of 20 of the dual edges (Fig. S2), when $p y k F$ was mutated. Examining the restoration of $p y k F$ (Fig. S3), only 3 of 22 edges changed significance and just one critical edge lost significance, indicating that the epistasis in this case occurs because the mutation causes new interactions. Thus, the $p y k F$ mutation appears to enable further evolution during the Lenski experiment (39) by distorting the epistatic landscape. $r b s$ also generates distortions (Fig. 2e), which can be visualized as a concentrated region of epistasis on the dual graph (Fig. 2e Inset). We found similar features in another genetic data set for the $\beta$-lactamase enzyme (44) (Appendix B7). Filtrations can thus reveal the specific geometric structure of both the interactions and the context they rely upon.

\subsection{Lactobacilli produce microbiome distortions}

Up to this point, we have focused on genetic epistasis, but our framework is generalizable to interactions of environmental parameters, including the gut microbiome, for which a framework to identify complex interactions is greatly needed. Like the genome, which is composed of many genes that interact to determine organismal fitness, the microbiome is also composed of many smaller units (bacterial species in this case) that affect host fitness. Hosts are known to select and maintain a certain core set of microbes $(45,46)$; the interactions of these bacteria can affect host fitness (3); and it is debated to what extent these interactions are of higher-order, c.f. (28). While vertebrates have a gut taxonomic diversity of $\approx 1000$ species, precluding study 
(a) Experimental design

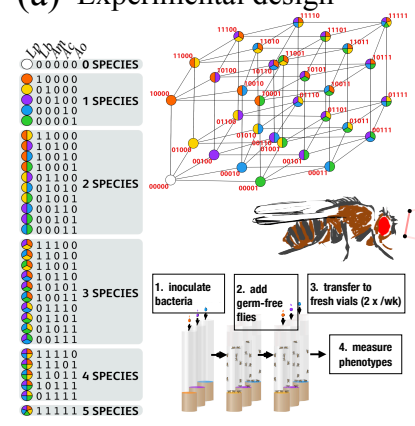

(c) filtration of $1^{* * * *}$

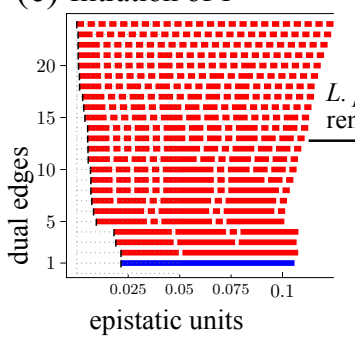

(b) graph of $* * * * *$

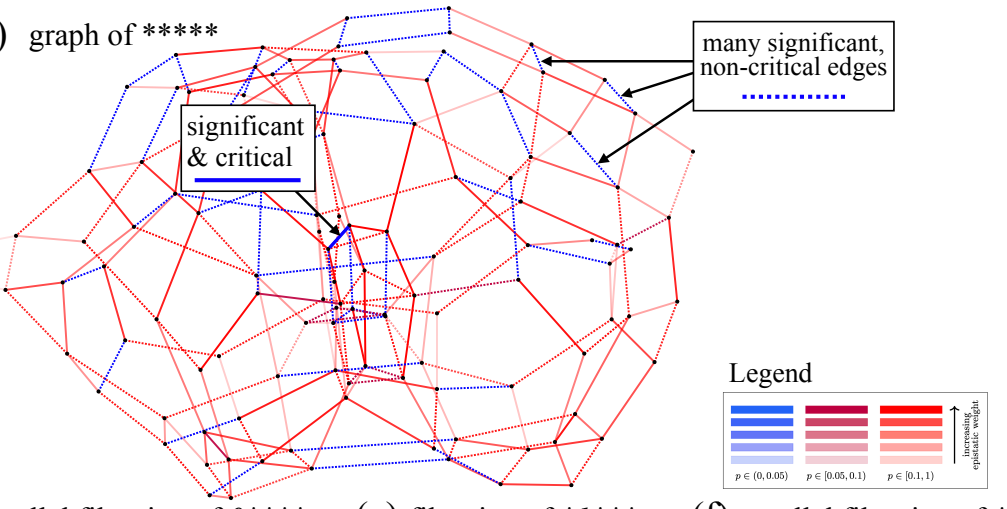

(d) parallel filtration of $0 * * * *$

(e) filtration of $* 1 * * *$

(f) parallel filtration of $* 0 * * *$

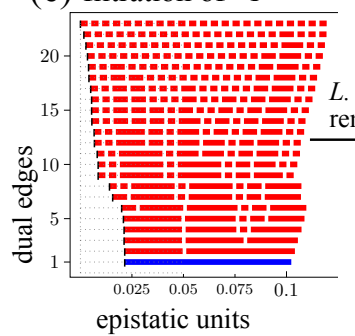

Figure 3: Loss of lactobacilli causes global distortion of the microbiome epistastic landscape. (a) Experimental design for Eble and Gould (3) microbiome manipulations in flies. (b) Full graph of $* * * * *$ for the Eble data. (c) Filtration of $\mathcal{S}(h)$ for the 4-face, $1 * * * *$, of Eble data, where L. plantarum is present, indicates epistasis where two clusters of maximal cells merge. (d) Parallel filtration with $L$. plantarum removed shows a landscape distortion. (e) Filtration for $* 1 * * *$, where L. brevis is present has similar structure to $1 * * * *$. (f) Parallel filtration with L. brevis removed shows a landscape distortion.

Epistasis was concentrated at the center of the dual graph (Fig. 3b,c), with significant, non- 
critical edges distributed throughout the graph (Fig. 3c). Examining the parallel transport, we found that the Lactobacilli drive changes in the global structure (Fig 3d,e). In 46 out of 128 (36\%) interactions, significance changed due to adding or removing a Lactobacillus (Fig 3cf, S7, S8). These changes in significance primarily derive from non-significant interactions when $L$. brevis is present that become significant when it is removed and vice versa, indicating L. brevis suppresses epistatic interactions that affect fly lifespan.

Microbiome abundances could drive the effects on host lifespan, however, comparing the epistatic landscapes for CFUs and lifespan, we found that only 2 of 99 dual edges were significant for both the bacterial abundance and fly lifespan data sets (Fig. S9, S10, S11, S12, Tables S2, S3, S4, S5), and there was a lack of correlation between the epistatic weights of the bipyramids (Spearman rank correlations: $p=0.7, p=0.5, p=0.3$, and $p=0.3$ respectively). This discord between the epistatic landscapes for microbiome fitness and host fitness could e.g. diminish the rate of co-evolution.

\subsection{Interactions are sparse in higher-dimensions}

We used epistatic filtrations to systematically evaluate the prevalence of higher-order interactions as a function of the number of dimensions. Critical, significant, higher-order interactions were less frequent than pairwise interactions $\left(p<10^{-6}, Z\right.$-test $)$ for each of the Khan, Eble, and Gould data sets, with a decreasing probability as a function of the face dimension (Table 1). This occurs for three primary reasons. First, the degrees of freedom increase in higher dimensions. Second, the probability of selecting a significant interaction from the set of all possible interactions decreases because the total number of interactions increases with increasing dimensions. Finally, the absolute number of significant interactions decreases in higher dimensions (Table 1), meaning they are biologically less prevalent. Overall, $\approx 10 \%$ of possible dual edges were significant at higher order, with $\approx 1 \%$ significant for $n=5$ dimensions (Table 1 ), 
suggesting limits to the dimensions of biological complexity.

We note that these fewer interactions in high dimensions can and do impact fitness. For example, the two top 4-dimensional interactions in the Eble microbiome data produce a combined 9\% effect on fitness (see edges 1 and 2 in (Fig. 3)) with the largest maximal cell accounting for $\approx 5 \%$. The relative sparsity makes for a tractable number of these interactions, where we may eventually determine the mechanisms, and filtrations provide a way to identify these.

Table 1: Prevalence of interactions at different levels of complexity in genetics and microbiome data sets. Significant versus all critical dual edges $(p<0.05)$.

\begin{tabular}{llll}
\hline Interaction dimension & Dataset: & $\begin{array}{l}\text { Dataset: } \\
\text { Eble }\end{array}$ & $\begin{array}{l}\text { Dataset: } \\
\text { Gould }\end{array}$ \\
\hline Khan & $20 / 80(25 \%)$ & $24 / 80(30 \%)$ & $22 / 80(28 \%)$ \\
all higher order: & $29 / 508(5.7 \%)$ & $58 / 540(10 \%)$ & $21 / 520(4.0 \%)$ \\
3: & $21 / 194(11 \%)$ & $35 / 199(17 \%)$ & $14 / 194(7.2 \%)$ \\
4: & $7 / 214(3.2 \%)$ & $22 / 226(10 \%)$ & $6 / 216(2.7 \%)$ \\
5: & $1 / 100(1.0 \%)$ & $1 / 115(0.8 \%)$ & $1 / 110(0.9 \%)$ \\
total: & $49 / 588(8.3 \%)$ & $82 / 620(13 \%)$ & $43 / 600(7.1 \%)$ \\
\hline
\end{tabular}

\subsection{Higher-order interactions can arise from lower-order interactions}

Non-linearities of lower-order interactions can produce interactions in higher dimensions (40). In examining the higher-order epistasis present in our data sets, we noted that the clusters where significant epistatic weights occur are often preceded by clusters with nearly significant epistatic weights in lower dimensions (Fig. S4). We developed a graphical approach to distinguish these interactions from those that arise de novo (Fig. S20b,c; Appendix B11).

Several higher-order interactions in the Gould and Khan data could not be attributed to lower-order effects (Table S6). In particular, they could not be detected from pairwise interactions between loci, (c.f. Fig. S20c). As we noted, the 4-dimensional interaction in the $E$. coli evolution experiment involved loci with two genes (Fig. 2), whereas in the microbiome, 
interactions involved loci with four species, indicating different underlying geometries at these different scales of biology (Table S6).

\section{Discussion and Conclusions}

From an evolutionary perspective, the Red Queen's hypothesis emphasizes how conflicts with other organisms can drive continuous genetic innovation (48). We find that epistasis in higher dimensions generates fitness landscape distortions, which could continuously change the fitness landscape to fuel new genomic innovation even in a static environment. This could partially explain the observation of continuous diversification in long term evolution experiments (49). In higher dimensions, we lack simple terminology to describe the many types of interactions that may occur, whether between quadruples and singles, pairs and triples, or different genetic backgrounds. We found that biologically-significant interactions in four and five dimensions are sparse and often rooted in lower order, meaning that a limited number of such interactions exist. This extends to higher dimensions the trend that 3-way interactions are often predicted from 2way interactions $(2,3,28)$. However, our finding that key genes and species cause distortions emphasizes the need to identify the significant higher-order interactions from the vast number of possible ones, a task that epistatic filtrations enable.

This geometric approach could be extended, e.g. to $\operatorname{GWAS}(15,19,50)$, ecosystems $(4,5)$, or neuronal networks (51), to discover non-additive higher-order structures at different scales. It should be noted that the polyhedral geometry methods for analyzing epistasis deserve to be developed further from the mathematical point of view. We believe that concepts of curvature for piecewise linear manifolds will be useful (52). 


\section{Acknowledgements}

The authors acknowledge L.J. Holt and O. Brandman for insightful comments on the manuscript. Research by M.J. is carried out in the framework of Matheon supported by Einstein Foundation Berlin. Further partial support by Deutsche Forschungsgemeinschaft (SFB-TRR 109: "Discretization in Geometry and Dynamics" and SFB-TRR 195: "Symbolic Tools in Mathematics and their Application". W.B.L. acknowledges NIH grant DP5OD017851, NSF IOS award 2032985, and the Carnegie Institution for Science Endowment.

\section{Competing interests}

The authors declare no competing interests.

\section{Supplementary Materials}

Materials and Methods

Fig S1 - S22

Tables S1 - S9

\section{References}

1. Daniel M Weinreich, Yinghong Lan, Jacob Jaffe, and Robert B Heckendorn. The Influence of Higher-Order Epistasis on Biological Fitness Landscape Topography. Journal of Statistical Physics, 172(1):208-225, feb 2018.

2. Elena Kuzmin, Benjamin VanderSluis, Wei Wang, Guihong Tan, Raamesh Deshpande, Yiqun Chen, Matej Usaj, Attila Balint, Mojca Mattiazzi Usaj, Jolanda Van Leeuwen, Elizabeth N Koch, Carles Pons, Andrius J Dagilis, Michael Pryszlak, Jason Zi, Yang Wang, 
Julia Hanchard, Margot Riggi, Kaicong Xu, Hamed Heydari, Bryan-joseph San Luis, Ermira Shuteriqi, Hongwei Zhu, Nydia Van Dyk, Sara Sharifpoor, Michael Costanzo, Robbie Loewith, Amy Caudy, Daniel Bolnick, Grant W Brown, Brenda J Andrews, Charles Boone, and Chad L Myers. Systematic analysis of complex genetic interactions. Science (New York, NY), 360(April 20), 2018.

3. Alison L. Gould, Vivian Zhang, Lisa Lamberti, Eric W. Jones, Benjamin Obadia, Nikolaos Korasidis, Alex Gavryushkin, Jean M. Carlson, Niko Beerenwinkel, and William B. Ludington. Microbiome interactions shape host fitness. Proceedings of the National Academy of Sciences, 115(51):E11951-E11960, 2018.

4. Ted J. Case and Edward A. Bender. Testing for Higher Order Interactions. The American Naturalist, 118(6):920-929, 1981.

5. Ian Billick and Ted Case. Higher Order Interactions in Ecological Communities : What Are They and How Can They be Detected? Ecology, 75(6):1529-1543, 1994.

6. Jacopo Grilli, György Barabás, Matthew J Michalska-Smith, and Stefano Allesina. Higherorder interactions stabilize dynamics in competitive network models. Nature, pages 1-5, jul 2017.

7. Casey Beppler, Elif Tekin, Zhiyuan Mao, Cynthia White, Cassandra McDiarmid, Emily Vargas, Jeffrey H. Miller, Van M. Savage, and Pamela J. Yeh. Uncovering emergent interactions in three-way combinations of stressors. Journal of the Royal Society Interface, 13(125), 2016.

8. Ivana Cvijović, Benjamin H. Good, Elizabeth R. Jerison, and Michael M. Desai. Fate of a mutation in a fluctuating environment. Proceedings of the National Academy of Sciences of the United States of America, 112(36):E5021-E5028, 2015. 
9. Lauren E. Nicolaisen and Michael M. Desai. Distortions in genealogies due to purifying selection and recombination. Genetics, 195(1):221-230, 2013.

10. Sewall Wright. The roles of mutation, inbreeding, crossbreeding and selection in evolution. Proceedings of the Sixth International Congress of Genetics, 1:356-366, 1932.

11. Michael Costanzo, Anastasia Baryshnikova, Chad L Myers, Brenda Andrews, and Charles Boone. Charting the genetic interaction map of a cell. Current opinion in biotechnology, 22(1):66-74, feb 2011.

12. Djordje Bajic, Jean C C Vila, Zachary D Blount, and Alvaro Sanchez. On the deformability of an empirical fitness landscape by microbial evolution. Proceedings of the National Academy of Sciences, 115(44):11286-11291, oct 2018.

13. John Maynard Smith. Natural selection and the Concept of a Protein Space. Nature, 225(February 7):563-564, 1970.

14. S Kauffman and S Levin. Towards a general theory of adaptive walks on rugged landscapes. Journal of Theoretical Biology, 128(1):11-45, sep 1987.

15. Örjan Carlborg and Chris S Haley. Epistasis: too often neglected in complex trait studies? Nature reviews Genetics, 5(8):618-625, aug 2004.

16. Jeremy Van Cleve and Daniel B. Weissman. Measuring ruggedness in fitness landscapes. Proceedings of the National Academy of Sciences of the United States of America, 112(24):7345-7346, 2015.

17. Michael Costanzo, Anastasia Baryshnikova, Jeremy Bellay, Yungil Kim, Eric D Spear, Carolyn S Sevier, Huiming Ding, Judice L Y Koh, Kiana Toufighi, Sara Mostafavi, Jeany Prinz, 
Robert P St Onge, Benjamin VanderSluis, Taras Makhnevych, Franco J Vizeacoumar, Solmaz Alizadeh, Sondra Bahr, Renee L Brost, Yiqun Chen, Murat Cokol, Raamesh Deshpande, Zhijian Li, Zhen-Yuan Lin, Wendy Liang, Michaela Marback, Jadine Paw, BryanJoseph San Luis, Ermira Shuteriqi, Amy Hin Yan Tong, Nydia van Dyk, Iain M Wallace, Joseph A Whitney, Matthew T Weirauch, Guoqing Zhong, Hongwei Zhu, Walid A Houry, Michael Brudno, Sasan Ragibizadeh, Balázs Papp, Csaba Pál, Frederick P Roth, Guri Giaever, Corey Nislow, Olga G Troyanskaya, Howard Bussey, Gary D Bader, Anne-Claude Gingras, Quaid D Morris, Philip M Kim, Chris A Kaiser, Chad L Myers, Brenda J Andrews, and Charles Boone. The genetic landscape of a cell. Science (New York, NY), 327(5964):425-431, jan 2010.

18. Sean R Collins, Kyle M Miller, Nancy L Maas, Assen Roguev, Jeffrey Fillingham, Clement S Chu, Maya Schuldiner, Marinella Gebbia, Judith Recht, Michael Shales, Huiming Ding, Hong Xu, Junhong Han, Kristin Ingvarsdottir, Benjamin Cheng, Brenda Andrews, Charles Boone, Shelley L Berger, Phil Hieter, Zhiguo Zhang, Grant W Brown, C James Ingles, Andrew Emili, C David Allis, David P Toczyski, Jonathan S Weissman, Jack F Greenblatt, and Nevan J Krogan. Functional dissection of protein complexes involved in yeast chromosome biology using a genetic interaction map. Nature, 446(7137):806-810, apr 2007.

19. Xuanyao Liu, Yang I Li, and Jonathan K Pritchard. Trans Effects on Gene Expression Can Drive Omnigenic Inheritance. Cell, 177(4):1022-1034.e6, may 2019.

20. Evan A Boyle, Yang I Li, and Jonathan K Pritchard. An Expanded View of Complex Traits: From Polygenic to Omnigenic . Cell, 169(7):1177-1186, jun 2017.

21. Juannan Zhou and David M. McCandlish. Minimum epistasis interpolation for sequencefunction relationships. Nature Communications, 11(1):1782, 2020. 
22. David M. McCandlish. Long-term evolution on complex fitness landscapes when mutation is weak. Heredity, 121(5):449-465, 2018.

23. Molly Schumer, Chenling Xu, Daniel L Powell, Arun Durvasula, Laurits Skov, Chris Holland, John C Blazier, Sriram Sankararaman, Peter Andolfatto, Gil G Rosenthal, and Molly Przeworski. Natural selection interacts with recombination to shape the evolution ofhybrid genomes. Science, 360(May 11):656-660, 2018.

24. Daniel M Weinreich, Yinghong Lan, C Scott Wylie, and Robert B. Heckendorn. Should evolutionary geneticists worry about higher-order epistasis? Current Opinion in Genetics \& Development, 23(6):700 - 707, 2013. Genetics of system biology.

25. Zachary R. Sailer and Michael J. Harms. High-order epistasis shapes evolutionary trajectories. PLOS Computational Biology, 13(5):1-16, 052017.

26. Michael J. McDonald, Daniel P. Rice, and Michael M. Desai. Sex speeds adaptation by altering the dynamics of molecular evolution. Nature, 531(7593):233-236, 2016.

27. Christoph Ratzke, Julien Barrere, and Jeff Gore. Strength of species interactions determines biodiversity and stability in microbial communities. Nature Ecology \& Evolution, pages 121, feb 2020.

28. Jonathan Friedman, Logan M Higgins, and Jeff Gore. Community structure follows simple assembly rules in microbial microcosms. Nature Publishing Group, 1:1-7, mar 2017.

29. Deepika Sundarraman, Edouard A Hay, Dylan M Martins, Drew S Shields, Noah L Pettinari, and Raghuveer Parthasarathy. Quantifying multi-species microbial interactions in the larval zebrafish gut. bioRxiv, pages 1-23, may 2020. 
30. Philippe Piccardi, Björn Vessman, and Sara Mitri. Toxicity drives facilitation between 4 bacterial species. Proceedings of the National Academy of Sciences of the United States of America, 2019.

31. Alicia Sanchez-Gorostiaga, Djordje Bajić, Melisa L. Osborne, Juan F. Poyatos, and Alvaro Sanchez. High-order interactions distort the functional landscape of microbial consortia. PLoS Biology, 17(12):1-34, 2019.

32. Heather J. Cordell. Detecting gene-gene interactions that underlie human diseases. Nature Reviews Genetics, 10(6):392-404, 2009.

33. Niko Beerenwinkel, Lior Pachter, and Bernd Sturmfels. Epistasis and shapes of fitness landscapes. Statist. Sinica, 17(4):1317-1342, 2007.

34. Amy Berrington de González and D. R. Cox. Interpretation of interaction: A review. Ann. Appl. Stat., 1(2):371-385, 122007.

35. Jakub Otwinowski and Joshua B. Plotkin. Inferring fitness landscapes by regression produces biased estimates of epistasis. Proceedings of the National Academy of Sciences of the United States of America, 111(22), 2014.

36. Ingileif B Hallgrimsdottir and Debbie S Yuster. A complete classification of epistatic twolocus models. BMC Genetics, 9(1):17, jan 2008.

37. Aisha I. Khan, Duy M. Dinh, Dominique Schneider, Richard E. Lenski, and Tim F. Cooper. Negative epistasis between beneficial mutations in an evolving bacterial population. Science, 332(6034):1193-1196, 2011. 
38. Holger Eble, Michael Joswig, Lisa Lamberti, and William B. Ludington. Cluster partitions and fitness landscapes of the drosophila fly microbiome. Journal of Mathematical Biology, May 2019.

39. Jeffrey E. Barrick and Richard E. Lenski. Genome dynamics during experimental evolution. Nature Reviews Genetics, 14(12):827-839, 2013.

40. Zachary R Sailer and Michael J Harms. Detecting High-Order Epistasis in Nonlinear Genotype-Phenotype Maps. Genetics, 205(3):1079-1088, mar 2017.

41. Hiroshi C. Ito and Akira Sasaki. Evolutionary branching in distorted trait spaces. Journal of Theoretical Biology, 489:110152, 2020.

42. Sven Herrmann and Michael Joswig. Splitting polytopes. Münster J. Math., 1:109-141, 2008.

43. J. L. Gill. Effects of Finite Size on Selection Advance in Simulated Genetic. Australian journal of biological sciences, 18(1508):599-617, 1965.

44. Longzhi Tan, Stephen Serene, Hui Xiao Chao, and Jeff Gore. Hidden randomness between fitness landscapes limits reverse evolution. Phys. Rev. Lett., 106:198102, May 2011.

45. R E Ley, M Hamady, C Lozupone, P J Turnbaugh, R R Ramey, J S Bircher, M L Schlegel, T A Tucker, M D Schrenzel, R Knight, and J I Gordon. Evolution of Mammals and Their Gut Microbes. Science (New York, NY), 320(5883):1647-1651, jun 2008.

46. Alice Risely. Applying the core microbiome to understand host-microbe systems. Journal of Animal Ecology, 89(7):1549-1558, 2020.

47. William B. Ludington and William W. Ja. Drosophila as a model for the gut microbiome. PLOS Pathogens, 16(4):1-6, 042020. 
48. Leigh Van Valen. Molecular evolution as predicted by natural selection. Journal of Molecular Evolution, 3(2):89-101, 1974.

49. Benjamin H. Good, Michael J. McDonald, Jeffrey E. Barrick, Richard E. Lenski, and Michael M. Desai. The dynamics of molecular evolution over 60,000 generations. Nature, 551(7678):45-50, 2017.

50. Gang Fang, Wen Wang, Vanja Paunic, Hamed Heydari, Michael Costanzo, Xiaoye Liu, Xiaotong Liu, Benjamin Vandersluis, Benjamin Oately, Michael Steinbach, Brian Van Ness, Eric E Schadt, Nathan D Pankratz, Charles Boone, Vipin Kumar, and Chad L Myers. Discovering genetic interactions bridging pathways in genome-wide association studies. $\mathrm{Na}$ ture Communications, 10(4274):1-18, 2019.

51. Michael W. Reimann, Max Nolte, Martina Scolamiero, Katharine Turner, Rodrigo Perin, Giuseppe Chindemi, Paweł Dłotko, Ran Levi, Kathryn Hess, and Henry Markram. Cliques of neurons bound into cavities provide a missing link between structure and function. Frontiers in Computational Neuroscience, 11(June), 2017.

52. John M. Sullivan. Curvatures of smooth and discrete surfaces. In Discrete differential geometry, volume 38 of Oberwolfach Semin., pages 175-188. Birkhäuser, Basel, 2008.

53. Ewgenij Gawrilow and Michael Joswig. polymake: a framework for analyzing convex polytopes. In Polytopes_combinatorics and computation (Oberwolfach, 1997), volume 29 of DMV Sem., pages 43-73. Birkhäuser, Basel, 2000.

54. Daniel M Weinreich, Yinghong Lan, Jacob Jaffe, and Robert B Heckendorn. The influence of higher-order epistasis on biological fitness landscape topography. Journal of statistical physics, 172(1):208-225, 2018. 
55. Jesús A. De Loera, Jörg Rambau, and Francisco Santos. Triangulations, volume 25 of Algorithms and Computation in Mathematics. Springer-Verlag, Berlin, 2010. Structures for algorithms and applications.

56. Daniel M Weinreich, Nigel F Delaney, Mark A Depristo, and Daniel L Hartl. Darwinian evolution can follow only very few mutational paths to fitter proteins. Science (New York, NY), 312(5770):111-114, apr 2006.

57. Deepika Sundarraman, Edouard A. Hay, Dylan M. Martins, Drew S. Shields, Noah L. Pettinari, and Raghuveer Parthasarathy. Higher-order interactions dampen pairwise competition in the zebrafish gut microbiome. mBio, 11(5):1-15, 2020.

58. Kabir Husain and Arvind Murugan. Physical Constraints on Epistasis. Molecular Biology and Evolution, 2020.

59. Andrés Aranda-Díaz, Benjamin Obadia, Ren Dodge, Tani Thomsen, Zachary F. Hallberg, Zehra Tüzün Güvener, William B. Ludington, and Kerwyn Casey Huang. Bacterial interspecies interactions modulate $\mathrm{pH}$-mediated antibiotic tolerance. eLife, 9, 2020.

60. E. D. Weinberger. Fourier and taylor series on fitness landscapes. Biological Cybernetics, 65(5):321-330, 1991.

61. Gareth James, Daniela Witten, Trevor Hastie, and Robert Tibshirani. An Introduction to Statistical Learning: With Applications in R. Springer Publishing Company, Incorporated, 2014.

62. Niko Beerenwinkel, Lior Pachter, Bernd Sturmfels, Santiago F. Elena, and Richard E. Lenski. Analysis of epistatic interactions and fitness landscapes using a new geometric approach. BMC Evolutionary Biology, 7(1):60, Apr 2007. 
63. E. D. Weinberger. Fourier and taylor series on fitness landscapes. Biological Cybernetics, 65(5):321-330, 1991.

64. Hye-Yeon Lee, Shin-Hae Lee, Ji-Hyeon Lee, Won-Jae Lee, and Kyung-Jin Min. The role of commensal microbes in the lifespan of Drosophila melanogaster. Aging, 11(13):4611$4640,2019$. 


\section{Supplementary Materials for: High dimensional geometry of fitness landscapes identifies master regulators of evolution and the microbiome}

Holger Eble, ${ }^{1}$ Michael Joswig, ${ }^{1,2 *}$ Lisa Lamberti ${ }^{3,4}$, William B. Ludington ${ }^{5,6 *}$

${ }^{1}$ Chair of Discrete Mathematics/Geometry, TU Berlin, Germany

${ }^{2}$ MPI MiS Leipzig, Germany

${ }^{3}$ Department of Biosystems Science and Engineering, ETH Zürich, Basel, Switzerland

${ }^{4}$ SIB Swiss Institute of Bioinformatics, Basel, Switzerland

${ }^{5}$ Department of Embryology, Carnegie Institution for Science, USA

${ }^{6}$ Department of Biology, Johns Hopkins University, Baltimore, MD, USA

${ }^{*}$ To whom correspondence should be addressed;

E-mail: joswig@math.tu-berlin.de, ludington@carnegiescience.edu. 


\section{A Materials and Methods}

\section{A.1 Fly husbandry}

Flies were reared germ-free and inoculated with one combination of bacteria on day 5 after eclosion. $N \geq 100$ flies were assayed for lifespan in $n \geq 5$ independent vials per bacterial combination for a total of 3200 individual flies. Food was $10 \%$ autoclaved fresh yeast, $5 \%$ filter-sterilized glucose, $1.2 \%$ agar, and $0.42 \%$ propionic acid, $\mathrm{pH} 4.5$. Complete methods are described in Gould et al (3).

\section{A.2 Bacterial cultures}

Bacteria were cultured on MRS or MYPL, washed in PBS, standardized to a density of $10^{7}$ $\mathrm{CFU} / \mathrm{mL}$ and $50 \mu \mathrm{L}$ was inoculated onto the fly food. Strains are indicated in Table S7. See Gould et al (3) for complete methods.

\section{A.3 Genetics data}

Existing genetics data sets were gotten from Sailer and Harms 2017 (40) github repository (https://github.com/harmslab/epistasis) or from Tan et al (44).

For the Khan data in Fig. 2, the fitness function $h$ is defined for (b) by assigning the following normalized values to the 16 genotypes:
$00000 \mapsto 0.1524 \quad 01000 \mapsto 0.1745 \quad 00100 \mapsto 0.1689 \quad 00010 \mapsto 0.1569$
$00001 \mapsto 0.1528 \quad 01100 \mapsto 0.1842 \quad 01010 \mapsto 0.1756 \quad 01001 \mapsto 0.1823$
$00110 \mapsto 0.1718 \quad 00101 \mapsto 0.1810 \quad 00011 \mapsto 0.1642 \quad 01110 \mapsto 0.1836$
$01101 \mapsto 0.1956 \quad 01011 \mapsto 0.1858 \quad 00111 \mapsto 0.1813 \quad 01111 \mapsto 0.1987$.

The Tan data set is different from the other fitness values in that only median and mean values are given, meaning we cannot compute $p$-values to assess the statistical significance. The fitness values are minimum inhibitory concentrations of antibiotics from a well-standardized assay with little experimental variation. Thus, the measurements and our analysis are believed to 
be robust. We note that the regular subdivision resulting from the corresponding height function of $[0,1]^{5}$ is degenerate in the sense that it is not a triangulation. This degeneracy arises because the data are discrete antibiotic concentrations with 24 possible values. The repetition of exact values in several cases means a triangulation does not occur. We extended our methods to this degenerate case by restricting the analysis to the faces that do have a triangulation, broadening the application of our approach. We focused on the piperacillin with clavanulate data from (44) as it is the better behaved.

\section{A.4 Computational analysis}

The filtrations code is available as a polymake (53) package (cf. https://github. com/holgereble/EpistaticFiltration) and the analysis pipeline is available as a jupyter notebook.

\section{B Terminology}

Loci (singular locus) refer to individual sites in the genome where a mutation may occur, or in the microbiome sense, a locus is a particular bacterial species. We write $[n]:=\{1, \ldots, n\}$ for the set of all loci.

Genotypes, $v=\left(v_{1}, \ldots, v_{n}\right)$, are vectors of loci with $0 / 1$-coordinates that form points in some fixed Euclidean space $\mathbb{R}^{n}$, where $n$ is the number of genetic loci or bacterial species considered. In this article we focus on biallelic $n$-locus systems, i.e. genotype sets of the form $V=\{0,1\}^{n}$ where $n$ is the number of loci and each locus is either 0 , absent, or 1 , present. For instance, $v=(1,0,1)$ denotes a genotype in a 3-locus system $\mathbb{R}^{3}$, where the first and third loci are mutant and the second is wild type. The set of all genotypes will be denoted by $V$. The convex hull $P:=\operatorname{conv}(V)$ of all genotypes is called the genotope. In our setting $P$ is the $n$-dimensional unit cube $[0,1]^{n}$ (c.f. (Fig. S21) for a $2 D$ projection of $[0,1]^{5}$ ). 
A fitness function (also called height function) associates to each genotype $v \in V$ a quantified phenotype describing the impact of the genotype on the organism. For example, if the measured phenotype is fitness, $h$ encodes the reproductive output of the genotype.

The fitness landscape is the pair $(V, h)$, which defines the fitness $h(v)$ for each genotype $v \in V$. Let $v=\left(v_{1}, \ldots, v_{n}\right) \in V$ be a genotype. Then its lift is given by $(v, h(v))=$ $\left(v_{1}, \ldots, v_{n}, h(v)\right) \in \mathbb{R}^{n+1}$.

A set of points $W=\left\{w^{(1)}, \ldots, w^{(\ell)}\right\}$ is affinely independent if for all real scalars $\lambda_{i}$ satisfying $\sum_{i=1}^{\ell} \lambda_{i}=0$ the condition $\sum_{i=1}^{\ell} \lambda_{i} w^{(i)}=0$ forces $\lambda_{i}=0$ for all $i \in\{1, \ldots, \ell\}$. Otherwise $W$ is affinely dependent.

An interaction with respect to a fitness function $h$ occurs between a collection of $k+2$ affinely dependent genotypes $v^{(1)}, \ldots, v^{(k+2)} \in V \subset \mathbb{R}^{n}$, for $k \leq n$, whose lifts are affinely independent points in $\mathbb{R}^{n+1}$. This is in line with the standard concept of additive epistasis. The number $k$ is the dimension of the interaction; throughout we assume that $k \geq 2$.

Let $U=\left\{v^{(1)}, \ldots, v^{(\ell)}\right\}$ be a set of genotypes. Its support is the set

$$
\operatorname{supp}(U):=\left\{k \in[n] \mid \text { there are distinct } 1 \leq i, j \leq \ell \text { with } v_{k}^{(i)} \neq v_{k}^{(j)}\right\}
$$

That is, the support is the set of loci where at least two of the given genotypes differ. For example, if $n=3$ and $U=\{(0,0,0),(1,0,1),(1,0,0)\}$ then $\operatorname{supp}(U)=\{1,3\}$.

The number of loci that vary ( 0 vs 1 ) in the support is called the order of an interaction; this definition agrees with, c.f., (54): "We designate interactions among any subset of $k$ mutations as $k$ th-order epistasis.". We give two examples: First, let $n=2$ and $U=$ $\{(0,0),(0,1),(1,0),(1,1)\}=V$ such that $U$ is an interaction with respect to some fitness function. Then $U$ is an interaction of dimension 2 and order 2. Second, let $n=3$ and $U=\{(0,0,0),(0,1,1),(1,0,0),(1,1,1)\}$ such that, again, $U$ is an interaction with respect to some height function. Then the dimension is 2 and the order is 3 . In general, the order is at least 
as large as the dimension, but the two quantities may differ. We say that genes (corresponding to loci) interact if they form the support set of an interaction of genotypes.

Remark. The dimension $k$ of an interaction $v^{(1)}, \ldots, v^{(k+2)}$ with respect to some fitness function agrees with the dimension of the affine span of the given points in $\mathbb{R}^{n}$. This can be seen as follows. By definition the lifted points $\left(v^{(1)}, h\left(v^{(1)}\right)\right), \ldots,\left(v^{(k+2)}, h\left(v^{(k+2)}\right)\right)$ are affinely independent in $\mathbb{R}^{n+1}$. So their affine span has dimension $k+1$. As $v^{(1)}, \ldots, v^{(k+2)}$ are affinely dependent, the dimension of their affine span is at most $k$. Now the affine dimension can only increase by at most one if one coordinate is appended.

\section{B.1 A primer on epistatic filtrations}

We first explain the biallelic case with $n \geq 2$ loci. In the geometric framework (33), two interacting loci give rise to four possible genotypes, which form the vertices of a square and may be written as vectors of zeros and ones, indicating the absence ( 0 , wildtype) or the presence $(1$, mutant) of each locus respectively (Fig. 1b) $(33,38)$. The measured phenotypes lift the genotype vertices into 3 -space, and there is epistasis corresponding to the volume of the simplex enclosed by the lifted points (see blue simplex in Fig. 1b). Geometrically, the four genotypes involved are fully symmetric, meaning that the sign of the epistasis for $n=2$ is relative to the choice of a coordinate system. Thus, the sign of epistasis depends on which genotype is considered wildtype. By considering the simplex volume rather than the fold of the upper shell of the simplex, epistatic filtrations do not specify a sign and thus avoid this caveat. However, directionality is considered by parallel transport (see later section). Returning to our explanation, by taking the upper convex hull of all $2^{n}$ lifted points and projecting back onto the genotope $[0,1]^{n}$ we induce a subdivision $\mathcal{S}(h)$; cf. $(38,55, \S 2.1)$, into maximal cells (Fig. 1b). Generically, every maximal cell of $\mathcal{S}(h)$ is an $n$-dimensional simplex, which is the convex hull of $(n+1)$ affinely independent genotypes (Fig. 1c). Importantly, these $n$-dimensional simplices are the most elementary 
parts into which a fitness landscape can naturally be decomposed.

Our framework generalizes to higher dimensions through a geometric shape called a bipyramid, where two satellite vertices, each the apex of one pyramid, are joined to a common set of base vertices. The satellites correspond in the $2 D$ example (Fig. 1b) to 00 and 11 and the base to 10 and 01 . This is naturally associated with $\mathcal{S}(h)$, set up by the ridge (Fig. 1b). For an ordered sequence of $n+2$ genotypes $\left(v^{(1)}, v^{(2)}, \ldots, v^{(n+2)}\right)$ we let

$$
s=\operatorname{conv}\left\{v^{(1)}, \ldots, v^{(n+1)}\right\} \quad \text { and } \quad t=\operatorname{conv}\left\{v^{(2)}, \ldots, v^{(n+2)}\right\} .
$$

In other words, $s$ and $t$ form convex hulls. We call such a pair $(s, t)$ a bipyramid with vertices $v^{(1)}, v^{(2)}, \ldots, v^{(n+2)}$. Then we can find the volume of the lifted bipyramid by forming the $(n+$ $2) \times(n+2)$-matrix

$$
E_{h}(s, t):=\left(\begin{array}{cccccc}
1 & v_{1,1} & v_{1,2} & \ldots & v_{1, n} & h\left(v^{(1)}\right) \\
1 & v_{2,1} & v_{2,2} & \ldots & v_{2, n} & h\left(v^{(2)}\right) \\
\vdots & \vdots & \vdots & \vdots & \vdots & \vdots \\
1 & v_{n+2,1} & v_{n+2,2} & \ldots & v_{n+2, n} & h\left(v^{(n+2)}\right)
\end{array}\right),
$$

where $v_{i, 1}, v_{i, 2}, \ldots, v_{i, n}$ are the coordinates of $v^{(i)} \in \mathbb{R}^{n}$. The epistatic weight of the bipyramid $(s, t)$ is

$$
e_{h}(s, t):=\left|\operatorname{det} E_{h}(s, t)\right| \cdot \frac{\operatorname{nvol}(s \cap t)}{\operatorname{nvol}(s) \cdot \operatorname{nvol}(t)} .
$$

Here nvol denotes the dimensionally normalized volume. The quantity nvol $(s \cap t)$ is the relative $(n-1)$-dimensional normalized volume of the ridge of the bipyramid, given by the intersection $s \cap t=\operatorname{conv}\left(v^{(2)}, \ldots, v^{(n+1)}\right)$. We use the notation

$$
\left\{v^{(1)}\right\}+\left\{v^{(2)}, \ldots, v^{(n+1)}\right\}+\left\{v^{(n+2)}\right\}
$$

for the bipyramid $(s, t)$, where the first and last vertices are the satellites and the middle set forms the base. Now the $n+2$ genotypes of the bipyramid form an interaction of dimension $n$ when $e_{h}(s, t)>0$. 
In our regular triangulation $\mathcal{S}(h)$, the two $n$-dimensional simplices, $s$ and $t$, are adjacent because their intersection $s \cap t$ is a common face of dimension $n-1$.

\section{B.2 Constructing a filtration from the epistasis of adjacent simplices}

We visualize the topography of the epistatic landscape by forming a dual graph of $\mathcal{S}(h)$, where the nodes are the maximal simplices and adjacent simplices form the dual edges. A rugged path is one with more blue edges (Fig. 1d). To each such dual edge we associate an epistatic weight and a label (Fig. 1c, epistatic weights are in shades of blue and red, while labels are in black). In this way, we construct an epistatic landscape that corresponds to the underlying fitness landscape with the ruggedness specified along the dual graph. The epistatic filtration of $h$ (Fig. 1e) depicts the path from weakest to highest epistasis by merging adjacent simplices. These diagrams summarize the information contained in epistatic weights and dual graphs, and facilitate comparisons across data sets. But there is important new information contained in epistatic filtrations, which is not directly visible from the dual graph and its epistatic weights. Indeed, a step in the epistatic filtration merges adjacent simplices. We build the complete fitness landscape by stepwise merging of maximal cells, starting from the lowest epistatic weight and stepwise merging adjacent simplices to form a connected cluster c.f. (38). In this sense, epistatic filtrations encode a global notion of epistasis in higher dimensions by connecting adjacent bipyramids.

To see this, notice that each row of the diagram has a number of bars and a black leftmost line. In the top row the black line marks the epistatic weight of zero ( $x$-coordinate). Each bar is red and corresponds to one maximal simplex of $\mathcal{S}(h)$. In the second row (counting from the top), we see three things: (1) the value of the lowest epistatic weight moves the $x$-coordinate of the black line slightly to the right. (2) The two maximal simplices of $\mathcal{S}(h)$ corresponding to this epistatic weight are merged into one. These correspond to the two bars in the previous row 
above the new, longer bar in the row. The lengths of the other bars remain unchanged but are shifted horizontally by the epistatic weight in (1). (3) The statistical significance of the epistatic weight giving rise to the merging step, encoded by the colors of the bars; cf. Section B.4.

The merging procedure is then repeated for each pair of maximal simplices arising in each epistatic weight until one reaches the highest epistatic weight and the last maximal simplex of $\mathcal{S}(h)$ to be merged with the rest. In this way the indentation of the bar charts increases from top to bottom. The total width of the bars stays constant throughout.

Importantly, in the epistatic filtration diagram, not every merging step is displayed; e.g., in Fig. 1d there are fewer rows than dual edges in Fig. 1c. This is because some steps do not change the resulting fitness landscape (no actual new portion is merged to the previous one). The reported steps are only the ones increasing the connected components of the fitness landscape obtained from the previous merging steps. The epistatic weights corresponding to these steps are the edges in the dual graph which we call critical in $(38, \S .3 .2)$.

\section{B.3 Normalized epistatic weights}

To gain a perspective on the generality of higher-order interactions, it is desirable to compare epistatic landscapes. Different phenotypes have different metrics, making comparisons difficult for current approaches to epistasis. Filtrations are well-suited in this sense. Scaling the height function $h$ by a positive constant does not change the regular triangulation, and thus it does not change the dual graph. In order to compare different data sets, we scale the height function to Euclidean norm one. The epistatic weights are scaled accordingly. The resulting normalized epistatic weights are measured in epistatic units, giving a generalized metric for epistasis.

Measuring the effect of context on epistatic interactions is also desirable, e.g. to detect the marginal or conditional effects of a locus (37), and these are a natural feature of filtrations. If we fix some $k$ loci and let the remaining $n-k$ loci vary, we obtain a height function, which is 
restricted to a face of the genotope $[0,1]^{n}$. That face has $2^{n-k}$ vertices, and it is an isomorphic copy of the cube $[0,1]^{n-k}$. For instance, if $n=5$ and we fix the first and the fourth locus to 0 , we obtain a 3 -dimensional face, which we denote $0 * * 0 *$. That is, such a face is written as a string of $n$ symbols in the alphabet $\{0,1, *\}$, where 0 or 1 mark the fixed choices, and $*$ stands for variation. The number of $*$ symbols equals the dimension of the face. Triangulations, their dual graphs, epistatic weights, etc. are well-defined for height functions restricted to faces. This aspect of the theory allows the study of conditional epistatic effects.

\section{B.4 Statistics of epistatic weights}

We developed a statistical test to quantify the significance of an interaction associated with a fixed bipyramid; cf. $(38, \S 4.2)$. Here we assume that $h(v)$ is the mean value of the individual phenotype measurements for some number of replicated experiments for the fixed genotype $v$. To each dual edge we associate a $p$-value, which is independent of the epistatic weight normalization. If that $p$-value is below 0.05 we call that dual edge significant. It is useful to also consider $p$-values, which are slightly higher because one can use the shape of the landscape to identify interesting locations for further statistical analysis. To this end we call a dual edge semi-significant if $0.05 \leq p<0.1$.

While it may be possible that this approach misses some biologically relevant interactions (e.g. if they do not correspond to a bipyramid selected by our method), those interactions that we identify carry information that is robust and supported by a statistical model. The fact that not all possible interactions can be approached is an inevitable consequence of the higher dimensional nature of fitness landscapes, also reflected by a very high number of possible regular triangulations of $[0,1]^{n}$. That number equals 74 for $n=3$ and $87,959,448$ for $n=4$, whereas the precise numbers for $n \geq 5$ are unknown; cf. $(55, \S 6.3)$. Thus, filtrations use the data to greatly condense the number of possible interactions considered. 
The bar colorings in the filtrations of epistatic weights, as in (Fig. S4), reflect the outcome of multiple simultaneous statistical tests (one for each epistatic weight) (38).

Significant dual edges at $p<0.05$ are shown in blue, $0.05 \leq p<0.1$ in purple, and $p \geq 0.1$ in red.

It may happen that a triangulation has a significant dual edge, which is not critical, whence it does not show in the epistatic filtration. In that case the next critical dual edge becomes blue; so a filtration encodes all significant interactions found by our method.

Remark. By funneling the analysis through the concept of regular triangulations our approach pre-selects interactions, which are most relevant with respect to fitness $(38, \S 2.2)$. Via this major deviation from (33) we are able to detect interactions in many data sets, which are biologically plausible; this suggests strongly that our method is particularly good at avoiding false positives. Future work will investigate the relationship to other methods from statistics and signal processing. While most of this is beyond the scope of the present study, in Appendix B12 we offer a first step by comparing with traditional linear regression approaches.

\section{B.5 A synthetic experiment examining how epistatic weights change as a function of the interaction order}

Our method calculates significance of detected interactions and normalizes the epistatic weight to the volume of the unit cube of the same dimensionality. We used synthetic data to analyze the method performance. We first examined 468 synthetic filtrations over the 4-dimensional cube, producing 10011 critical dual edges. We found that the epistatic weight is indeed constant as a function of the interaction order, see (Fig. S19a). This indicates that the normalization method is effective. Furthermore, the number of significant interactions decreased as the standard deviation of the input data increased, indicating the statistical method is sensitive to noise, see (Fig. S19b). 


\section{B.6 A microbiome example in dimension 4}

Here $n=4$, and the fitness function $h$ is defined by assigning the following values to the 16 genotypes:

$$
\begin{aligned}
& 0000 \mapsto 0.2484 ; \quad 1000 \mapsto 0.2320 ; \quad 0100 \mapsto 0.1618 ; \quad 0010 \mapsto 0.1698 ; \\
& 0001 \mapsto 0.1943 ; \quad 1100 \mapsto 0.1749 ; \quad 1010 \mapsto 0.1714 ; 1001 \mapsto 0.1929 ; \\
& 0110 \mapsto 0.1668 ; 0101 \mapsto 0.1608 ; \quad 0011 \mapsto 0.1617 ; \quad 1110 \mapsto 0.1643 ; \\
& 1101 \mapsto 0.1677 ; \quad 1011 \mapsto 0.1715 ; \quad 0111 \mapsto 0.1613 ; \quad 1111 \mapsto 0.1594 \text {. }
\end{aligned}
$$

The vertices $U:=\left\{v^{(1)}, \ldots, v^{(6)}\right\} \in V$ given by

$$
\begin{aligned}
& v^{(1)}=(1,1,0,0) ; \quad v^{(2)}=(0,0,0,0) ; \quad v^{(3)}=(1,0,0,0) ; \\
& v^{(4)}=(1,1,0,1) ; \quad v^{(5)}=(1,1,1,1) ; \quad v^{(6)}=(1,0,0,1)
\end{aligned}
$$

form a bipyramid $(s, t)$ consisting of 4 -dimensional simplices $s$ and $t$ as above. The simplices $s$ and $t$ correspond to nodes in the dual graph of $\mathcal{S}(h)$ that share a dual edge recording their adjacency relation as indicated in (Fig. 3b).

In this situation, equation (4) reads

$$
e_{h}(s, t)=\left|\begin{array}{llllll}
1 & 1 & 1 & 0 & 0 & 0.1749 \\
1 & 0 & 0 & 0 & 0 & 0.2484 \\
1 & 1 & 0 & 0 & 0 & 0.2320 \\
1 & 1 & 1 & 0 & 1 & 0.1677 \\
1 & 1 & 1 & 1 & 1 & 0.1594 \\
1 & 1 & 0 & 0 & 1 & 0.1929
\end{array}\right| \cdot \frac{\operatorname{nvol}(s \cap t)}{\operatorname{nvol}(s) \cdot \operatorname{nvol}(t)}=0.0318 \cdot \frac{\sqrt{2}}{1 \cdot 1} \approx 0.045
$$

Since $e_{h}(s, t)>0$, the genotype set $U$ defines a 4-dimensional interaction with full support $\{1,2,3,4\}$ and of order 4 , according to our terminology of Section Terminology. With a $p$ value of $0.0005<0.05$ the significance test established in $(38, \S .4)$ rejects the zero hypothesis for $e_{h}(s, t)$ and therefore proves the effect of the interaction $U$ to be significant. We indicate this fact with the color blue both in the dual graph of $\mathcal{S}(h)$ in (Fig. 3b) and in the epistatic filtration of $h$ in (Fig. 3c).

This example illustrates the following fact of biological interest. For the bacterial combinations $v^{(1)}, v^{(2)}, \ldots, v^{(6)}$ fitness, given by the fitness function $h$, varies significantly in a 
non-linear way.

\section{B.7 The epistatic landscape within a single enzyme is rugged}

As a point of comparison with the Khan data set, we re-analyzed data from a fully factorial 5 -mutation data set in the $\beta$-lactamase gene, where each mutation is in a separate residue of the same enzyme $(44,56)$. Due to a lack of the raw replicate data, our computations are based on the reported mean values, and $p$-values are not calculated. The filtration holds a high magnitude of epistasis (Fig. S5, S6) compared with the Khan data set (Fig. S4, S2); note magnitude on the $x$-axis. The epistasis arises in many steps (note slope of filtration on left side; (Fig. S5, S6)), consistent with the low number of possible evolutionary paths observed by Weinreich (56), and distortions are apparent in the shifted magnitude of epistasis by parallel transport. Our geometric approach also reveals a tiered structure to the epistasis, c.f. the largest weight merges two clusters of simplices (Fig. S5, S6), indicating a more complex epistatic landscape than the Khan data set, where epistasis came from one individual simplex on the periphery of the dual graph.

Examining the filtration (Fig. 3d), the epistatic weight (i.e. magnitude) for the microbiome data generated $\approx 5 \%$ effect, roughly three times the weight in the Khan data and half that in the Tan $\beta$-lactamase landscapes (44) (c.f. $x$-axis between Fig. 3, S4, S5), indicating that the rugosity of microbiome interactions is comparable to genetic ones.

To further compare the global effect of context across different datasets, we developed a method to compute epistasis, based on the triangulation of dual landscapes, which we call the epistatic product [Appendix Product model for epistasic landscape rugosity] (Fig. S13, S14, $\mathrm{S} 15, \mathrm{~S} 16, \mathrm{~S} 17, \mathrm{~S} 18)$. The total epistasis was highest for the $\beta$-lactamase experiment (44), which carries much higher context-dependence than either the microbiome (3) or E. coli evolution data sets (37), indicative of overall high epistasis at the smallest, within enzyme, scale. 


\section{B.8 Interactions are sparse in higher-dimensions}

The prevalence and importance of higher-order interactions is debated, with some studies suggesting pairwise interactions predict the vast majority of interactions in complex communities (28), and others suggesting a large influence of context-dependent effects (3) (57), which would make higher-order interactions unpredictable. As we showed in the previous section, few such interactions are biologically meaningful in the context of fitness.

This limitation on epistasis in higher dimensions could arise due to e.g. limited phenotypic dimensions where interactions can be detected or to a lower dimensional manifold that absorbs the majority of the effects (58) (e.g. lifespan and fecundity are anti-correlated, making fitness robust to changes in one or the other). Regardless, our analysis shows that significant epistatic interactions are increasingly sparse as the number of dimensions for interaction increase, indicating there exist some limits to biological complexity.

We analyzed the few higher-order interactions in greater detail using a geometric approach. As we noted previously, the interactions in the Khan genetic data (Table 1) are based on a vertex split of the genotype 00001, meaning that the entire epistatic weight of the landscape is balanced by a single maximal cell (Fig. 2).

In contrast, the epistatic filtration of the Eble microbiome data in (Fig. 3) has a much richer texture. There are two significant bipyramids

$$
\begin{aligned}
& \{01001\}+\{00000,01000,01101,01111\}+\{01100\} \quad 0.0451 \# 2 \\
& \{01001\}+\{00000,01000,01011,01111\}+\{01110\} \quad 0.0485 \# 1
\end{aligned}
$$

given with their epistatic weights and edge id's, which form a cluster of interactions, indicating a larger topographic feature in the epistatic landscape that relates the interactions between L. brevis and increasing numbers of Acetobacters. Proximal to these significant cells are two cells with nearly significant statistical support:

$$
\begin{aligned}
& \{01011\}+\{00000,01001,00111,01111\}+\{01101\} \quad \# 8 \\
& \{01011\}+\{00000,01000,01001,01111\}+\{01101\} \quad \# 7
\end{aligned}
$$


with their edge id's (Fig. 3). This invites further research on the bacteria involved. For instance, the interactions could derive from metabolic crossfeeding between the Acetobacters, which produce many co-factors, and L. brevis, which produces lactate, stimulating Acetobacter growth (59). Note that the support sets of the bipyramids for all four interactions contain both the wild type 00000 and 01111 , which are the maximum and minimum fitness respectively.

\section{B.9 Parallel transport of epistatic weights}

The notion of parallel transport in a fitness landscape $(V, h)$ was introduced in $(38, \S 6.6)$ as a way to compare geometric and biological information between pairs of parallel facets of the convex polytope conv $V$. In this work, we extended that notion to include the case of two fitness landscapes, $\left(V, h_{1}\right)$ and $\left(V, h_{2}\right)$, associated to different generic and normalized height functions $h_{i}: V \rightarrow \mathbb{R}, i \in\{1,2\}$, defined on the same vertex set $V=\{0,1\}^{n}$ for some $n \in \mathbb{N}$. To enable meaningful comparisons, we assume that each $h_{i}$ is normalized and that there is a larger fitness landscape $(W, h)$ with a generic and normalized height function $h: W \rightarrow \mathbb{R}$ restricting to $h_{1}$ and $h_{2}$ on the parallel facets $V$ in $W$, such that the partition of conv $W$ induced by $h$ is compatible with the one of conv $V$ induced by $h_{1}$, resp. by $h_{2}$. In this setting, we define normalized epistatic weights as with Eq. (4) with $h$ the normalized height function and $s, t$ any adjacent simplices forming a bipyramid.

Parallel transports enable us to transport epistatic filtrations along the reflection map

$$
\phi: V \rightarrow V ; v=\left(v_{1}, v_{2}, \ldots, v_{n}\right) \mapsto\left(v_{1}^{\prime}, v_{2}^{\prime}, \ldots, v_{n}^{\prime}\right)
$$

with $v_{i}^{\prime}=1-v_{k}$ if $i=k$ and $v_{i}^{\prime}=v_{i}$ otherwise. More precisely, let $e_{h_{1}}(s, t)$ be the normalized epistatic weight associated to a bipyramid of $\mathcal{S}\left(h_{1}\right)$ and let $\phi\left(e_{h_{1}}(s, t)\right):=e_{h_{2}}(\phi(s), \phi(t))$ be the parallel normalized epistatic weight transported by $\phi$. Then the filtration of normalized epistatic weights induces a filtration of parallel normalized epistatic weights. Additionally, to $e_{h_{1}}(s, t)$ and to $\phi\left(e_{h_{1}}(s, t)\right)$ a $p$-value can unambiguously be associated (38, §4.1-4.2). Notice 
that by design epistatic filtrations for $\mathcal{S}\left(h_{1}\right)$ only show normalized epistatic weights associated to critical dual edges, defined as in (38). But normalized epistatic weights and their significance can be defined for all bipyramids including the ones associated to noncritical dual edges. This explains the labelling of the parallel transport tables below. There a row is numbered only if the bipyramid corresponds to a critical dual edge in the dual graph of $\mathcal{S}\left(h_{1}\right)$. Noncritical dual edges whose normalized epistatic weight remains non-significant after the parallel transport are omitted. The normalized epistatic weight before (denoted by $e_{o}=e_{h_{1}}(s, t)$ ) and after (denoted by $\left.e_{p}=\phi\left(e_{h_{1}}(s, t)\right)\right)$ the parallel transport, as well as their $p$-values (denoted by $p_{o}$ and $p_{p}$ ) are also reported, as well as ratios of these quantities.

These parallel transport tables are linked to the epistatic filtration diagrams. Indeed, each numbered row in the table corresponds to the row in the epistatic filtration diagram with the black line set at $e_{o}$. It also corresponds to the row with black line set at $e_{p}$ in the parallel transported filtration diagram.

Recall from Section Statistics of epistatic weights that there may be dual edges of the triangulations which are significant but not critical. Since only the critical dual edges are labeled (by the row number in the epistatic filtration), in our tables for parallel transport these show up as unlabelled rows.

Examples for the parallel transport of epistatic filtrations are shown in Figures S1, S2, $\mathrm{S} 3$, S5, and S6. The magnitude of the epistasis in the left panels are roughly comparable between data sets due to normalization of the input data. Compare each left panel with its corresponding right panel to observe the relative change in epistasis in the parallel path. Larger changes in epistasis indicate stronger context-dependence of the interaction. For instance, in the first Weinreich comparison (Fig. S5), bar 10 in the right panel has a parallel epistasis greater than the original filtration on the left, indicating context-dependence. 


\section{B.10 Product model for epistasic landscape rugosity}

In this section we offer a new methodological framework to simultaneously study fitness landscapes associated to different height functions. We also provide a measure to quantify how much the height function of the combined fitness landscape differs from the sum of the height functions.

Let $U$ and $V$ be point configurations in $\mathbb{R}^{m}$ and $\mathbb{R}^{n}$, respectively. We think of these point configurations as two sets of genotypes, which may be distinct or not. If we have height functions $\lambda: U \rightarrow \mathbb{R}$ and $\mu: V \rightarrow \mathbb{R}$, then taking the sum $\lambda+\mu$ point-wise yields a lifting function of the product $U \times V \subset \mathbb{R}^{m+n}$. The cells of the regular subdivision $\mathcal{S}_{U \times V}(\lambda+\mu)$ are products of cells of $\mathcal{S}_{U}(\lambda)$ with cells of $\mathcal{S}_{V}(\mu)$. In particular, if $\lambda$ and $\mu$ are generic, i.e., $\mathcal{S}_{U}(\lambda)$ and $\mathcal{S}_{V}(\mu)$ are triangulations, then the cells of $\mathcal{S}_{U \times V}(\lambda+\mu)$ are products of simplices.

Now we consider an arbitrary height function $\nu: U \times V \rightarrow \mathbb{R}$ on the product of the point configurations. This yields height functions

$$
\nu_{U}: U \rightarrow \mathbb{R}, u \mapsto \frac{1}{\ell} \sum_{v \in V} \nu(u, v) \quad \text { and } \quad \nu_{V}: V \rightarrow \mathbb{R}, v \mapsto \frac{1}{k} \sum_{u \in U} \nu(p, q),
$$

where $k=\# U, \ell=\# V, u$ is a vertex in $U$ and $v$ is a vertex in $V$.

Further we define

$$
\nu^{\prime}: U \times V,(u, v) \mapsto \nu(u, v)-\nu_{U}(u)-\nu_{V}(v)
$$

Observe that

$$
(\lambda+\mu)_{U}(u)=\lambda(u)+\frac{1}{\ell} \sum_{v \in V} \mu(v) \text { and }(\lambda+\mu)_{V}(q)=\mu(v)+\frac{1}{k} \sum_{u \in U} \lambda(u),
$$

and $(\lambda+\mu)^{\prime}$ is the height function with constant value $-\left(\frac{1}{k} \sum_{u \in U} \lambda(u)+\frac{1}{\ell} \sum_{v \in V} \lambda(v)\right)$. Thus $\lambda+\mu$ and $(\lambda+\mu)_{U}+(\lambda+\mu)_{V}$ induce the same regular subdivision of $U \times V$. Therefore, we propose to analyze the height function $\nu^{\prime}$ to measure how much $\nu$ deviates from the sum of 
two height functions. We can use the techniques from our previous paper (38) and apply (all of) them to $\mathcal{S}_{U \times V}\left(\nu^{\prime}\right)$ for any given $\nu$. For instance, this allows to measure how independent two different height functions are on the same point set (this is the case $U=V$ ). We say that $\nu$ decomposes as a product if $\nu^{\prime}=0$.

Example 1. If $U=V=\{0,1\}$ are the vertices of the unit interval then $U \times V$ are the vertices of the unit square $[0,1]^{2}$. Analyzing $\mathcal{S}\left(\nu^{\prime}\right)$ for any given height function $\nu$ on the four points $(0,0),(0,1),(1,0)$ and $(1,1)$ gives back the standard basic example of additive epistasis.

Remark. Two observations are in order: In $(38, \S 6.6)$ we considered a version of parallel transport to compare epistatic effects, see also Appendix B9. The connection to the product model approach is as follows. Let $V=\{0,1\}^{n}$, i.e., the vertex set of the $n$-dimensional unit cube, be embedded twice, into a pair of parallel facets of the unit $(n+1)$-cube $[0,1] \times[0,1]^{n}$. This occurs in the product model with $U=\{0,1\}$. If a height function $\nu$ on $\{0,1\} \times U$ decomposes as a product then the parallel transport (in both directions) is trivial. Note that the number of dimensions is greater for the product model than for the parallel transport.

Additionally, observe that the product model differs from the marginal epistasis framework, which would produce a single number testing if the mutant changes one specific interaction between the genes.

\section{B.10.1 Product model for the Khan data}

To illustrate the product model consider the following example from the Khan data. We are interested in detecting if interactions between the topA, spoT, and $p y k F$ genes change when the $r b s$ gene is mutated. To answer this question we let $U$ and $V$ be 3 -cubes inside $[0,1]^{5}$ defined by three mutable loci, one for each of the above genes and indicated by $*$, and two fixed loci. The first fixed locus represents the $r b s$ gene. It is not mutated in $U$ and mutated in $V$. The height functions are compared over the three variable loci. Thus the filtration over the product 
model for $U$ and $V$ has four dimensions in this case. A computation reveals that there are no significant dual edges in the epistatic filtration on product model, see (Fig. S13). This indicates that the $r b s$ mutant does not affect the interaction landscape.

\section{B.11 Meta-epistatic charts}

This section deals with the question to which extent higher order epistatic effects are induced by lower dimensional ones or, put in other terms, which lower dimension epistatic effects can be seen in higher dimension. The meta-epistatic chart is a diagram drawn on top of the induced epistatic filtrations for some selection of faces of a fixed cube; higher-order interactions induced by lower order interactions are marked as corresponding.

In (Fig. S20b) and (Fig. S20c) we exhibit an example for the Eble data set, with 5 loci, where we take the five 4 -dimensional faces $0 * * * *, * 0 * * *, * * 0 * *, * * * 0 *$ and $* * * * 0$ into consideration. Mathematically, these five 4-faces constitute the face figure of the wild type. Fix one 4-face, say $0 * * * *$. The induced epistatic filtration on this face shows two blue bars corresponding to dual edges labeled 1 and 2. Each of them refers to the ridge of a bipyramid, which is a 3-dimensional simplex in this case. These two ridges may intersect certain 3-dimensional faces in the right dimension and thus may or may not descend to significant ridges within certain 3-dimensional filtrations. In case of an incidence with a lower dimensional significant ridge, the significant 4-dimensional effect is induced by a lower dimensional effect and one may picture this fact as a directed assignment pointing from the lower towards the higher dimensional interaction.

\section{B.12 Comparison with a simple linear regression approach}

In the theory of fitness landscapes many linear regression approaches have been proposed to study higher-order interactions, c.f. $(21,34,40,60)$. In this section, we compare our epistatic weight method to an elementary regression approach using an example from the data. 
The regression analysis we have in mind assumes that there is a linear relationship between the predictors $X_{1}, X_{2}, \ldots, X_{n}$ (one associated to each locus/dimension of the genotope) and response, or dependent, variables $Y$ (associated to the biological measurements). That is, one assumes that $Y=f\left(X_{1}, X_{2}, \ldots, X_{n}\right)+\epsilon$ where $f: \mathbb{R}^{n} \rightarrow \mathbb{R} ;\left(X_{1}, X_{2}, \ldots, X_{n}\right) \mapsto \beta_{0}+$ $\beta_{1} X_{1}+\beta_{2} X_{2}+\cdots+\beta_{n} X_{n}$ and where $\epsilon$ is a random error term. The coefficients $\beta_{1}, \beta_{2}, \ldots, \beta_{n}$ are unknown but can be estimated by minimizing the sum of squared residuals associated to the observations pairs $(x, y)$. These observations pairs consisting of a genotype and a measurement associated to it. Notice that more than one measurements are typically associated to a single genotype. With the coefficient estimates one can make predictions for the dependent variable via

$$
\hat{y}=\hat{\beta}_{0}+\hat{\beta}_{1} x_{1}+\hat{\beta}_{2} x_{2}+\cdots+\hat{\beta}_{n} x_{n}
$$

The hat symbol ^ indicates a prediction, for instance of $Y$ on the basis of $x_{i}=X_{i}$, or an estimate for an unknown coefficient.

Below, we are interested in the differences between the observed measurements $y$ associated to the genotypes of $[0,1]^{n}$, expressed in terms of $x_{1}, x_{2}, \ldots x_{n}$ and the predicated values $\hat{y}$ on the regression hyperplane (6). Notice that the regression analysis remains unchanged after normalizing the height function to Euclidean norm one. Additionally, computing residues for all replicated measurements (when provided) and then take averages builds on the assumption that measurements associated to different genotypes are statistically independent from each other. This assumption is consistent with the one underlying the computation of statistical significances for epistatic weights, following (38, §. 4.2-4.3).

Remark. In the regression setting of (6) there are hypothesis tests (like the $F$-statistic, $t$ statistics and $p$-value) to answer if at least one regression coefficient $\beta_{j}, 1 \leq j \leq n$ is nonzero, see for example (61). Such statistical approaches are different from the one in (38, §. 4.2-4.3), where other hypothesis tests for each epistatic weight were proposed. 


\section{B.12.1 Regression for Eble data}

In the following, we perform a regression analysis focusing on the replicated measurements for the lifespan fitness landscape on $[0,1]^{5}$ obtained from Eble and subspaces thereof. Numerical measures of model fit ( $F$-statistic: 2357, with $p$-value essentially zero, and for 3840 observations and 5 predictors) show that the multiple linear regression model can be considered to be appropriated for this data. Since the epistatic weights of the dual edges are close to zero $(\leq 0.02)$ and are mostly not significant, the above regression analysis conclusion is in line with what we see from the filtration of epistatic weights associated to the same fitness landscapes, see (Fig. S22).

From this example we see that the regression approach provides some general information on higher-order interactions. However, without further assumptions, only one interaction formula is given in terms of a regression hyperplane (6) while the epistatic weight approach gives more fine grained information. This example also illustrate that when the regression model fits the data well (essentially the higher the $F$-statistics and the more coefficients in the hyperplane equation are significantly non-zero) the epistatic filtration has little horizontal shifts and few significant epistatic weights.

We now proceed repeating the above analysis on some of the bipyramids considered in the parallel analysis for the normalized lifespan Eble data. Regressing over bipyramid 23 in Table S8

$$
\{0001\}+\{0000,1001,1011,0111\}+\{1111\}
$$

in $0 * * * *$ and $1 * * * *$ reveals that only two average residues over $0 * * * *$ are non-zero (associated to the microbiomes 00000 and 00001 ), and only one is non-zero over $1 * * * *$ (associated to the microbiome 10000). This confirms the two non significant epistatic weights over bipyramid 23 in Table S8. 
Remark. If minimally dependent sets of points in the genotope are fixed, as in the epistatic weight approach, and one regresses above these points, then the corresponding regression hyperplanes equations are learned from data and the equations generally differ from the epistatic weights given as in (4), but similar biological and geometric conclusions can be drawn. This idea could then be taken further by considering smoothing splines, instead of linear regression, and their relation to epistatic filtrations. From an application point of view, one would obtain an interesting new extension of the concept of epistasis because intermediate genotypes could be assessed, which would correspond to the case of genetically heterogeneous populations of organisms as occur in nature.

Other numerical results for the above regressions are summarized in Table S9. Over $0 * * * *$ two coefficients are significantly non-zero (for $x_{1}$ and $x_{4}$ ), see top part of Table S9. Similarly, over $1 * * * *$ four coefficients are significantly non-zero $\left(x_{1}, x_{2}, x_{3}, x_{4}\right)$, see bottom part of Table S9. The fit of the linear regression models is confirmed by the relatively high values of the $F$-statistic. Over $0 * * * v$ the $F$-statistics is 459.1 for a $p$-value near zero and 720 observations. Over $1 * * * *$ the corresponding $F$-statistics (near zero) is 52.61 .

\section{B.13 Comparison with other approaches}

Currently the main lines of research to investigate higher-order epistasis in computational biology and related disciplines include the present methods, inspired from discrete polyhedral geometry $(3,33,38,62)$; linear regression approaches, c.f. $(21)$; methods originating from harmonic analysis, c.f. $(40,54,63)$; and using correlations between the effects of pairwise mutations, discussed in (38).

In a 2-locus, biallelic system, all these methods can easily be recovered from one another; some of them even agree. This is true also for some ecological approaches, including the generalized Lotka-Voleterra equations, which yield a mathematically equivalent form to epistasis for 
certain situations c.f. see equation 9 of (4). In higher dimensional systems, these methods remain conceptually closely related but they generally yield different insights about the problem, such as whether the interactions are significant, what their magnitude is, and what their sign is. Because these previous methods make specific, a priori assumptions about the forms of interactions, they are limited by these assumptions. Epistatic filtrations add a global perspective, determining the structure of interactions from the shape of the fitness landscape.

\section{B.14 Microbiome data sets}

In this work, Drosophila microbiome fitness landscapes consist of experimental measurements on germ-free Drosophila flies inoculated with different bacterial species. The lifespan of approximately 100 individual flies were measured for each combination of bacterial species, giving roughly 3,200 individual fly lifespans for each of the two data sets presented. The experimental methods are described in $(3,64)$. The first data set is the exact data presented in $(3,64)$. The second data set is the second set of species with exactly the same methods used in $(3,64)$. The bacterial compositions considered consist of all possible combinations of five species. The species considered can all occur naturally in the gut of wild flies: Lactobacillus plantarum (LP), Lactobacillus brevis (LB), Acetobacter pasteurianus (APa), Acetobacter tropicalis (AT), Acetobacter orientalis (AO), Acetobacter cerevisiae (AC), Acetobacter malorum (AM). The 5member communities both stably persist in the fly gut. For the purposes of this work, we define stable as maintaining colonization of the gut when $\leq 20$ flies are co-housed in a standard fly vial and transferred daily to fresh food containing $10 \%$ glucose, $5 \%$ live yeast that has subsequently been autoclaved, $1.2 \%$ agar, and $0.42 \%$ propionic acid, with a $\mathrm{pH}$ of 4.5 . The total number of species found stably associated with an individual fly is typically between 3 and 8 . Consistently, Lactobacillus plantarum and Lactobacillus brevis, are found with two to three Acetobacter species. Less consistently, species of Enterobacteria and Enterococci occur, and 
838 these have been described as pathogens. While more strains may be present, for each of the two 839 data sets in the present work, a set of five non pathogen species was chosen, including the two 840 Lactobacilli and three Acetobacter species. The combinations of species are shown in Table S7. 841 Different strains of the same species were used in the two data sets. 
(a)

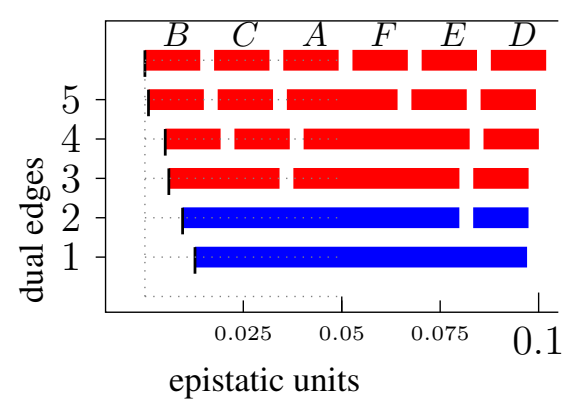

(b)

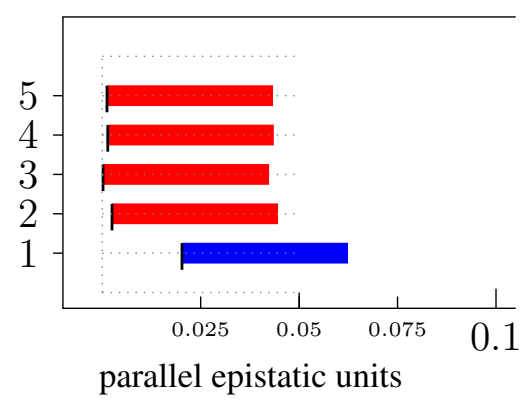

(c)
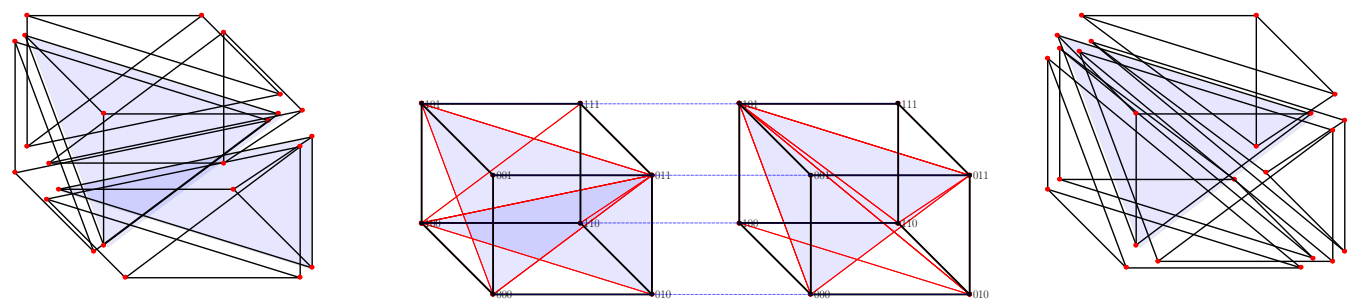

Figure S1: Parallel transport from $0 * * 0 *$ to $1 * * 0 *$ within the Khan dataset. (a) Filtration based on the triangulation of $0 * * 0 *$. (b) Parallel epistatic weights computed from $1 * * 0 *$ for the triangulation based on $0 * * 0 *$. (c) The two parallel triangulations (and exploded copies) are depicted. The partitions in the node set are transferred from the cube on the middle left to the cube on the middle right. Exploded versions of these same triangulation on the far left and far right demonstrate the geometry of the simplices generated by the triangulations. 
(a)

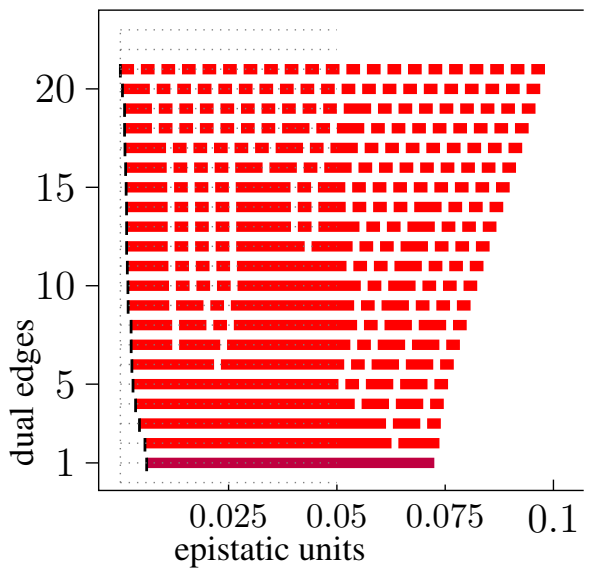

(b)

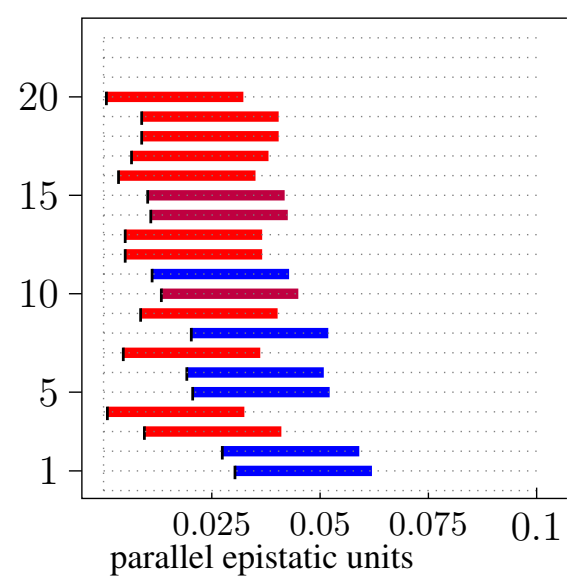

Figure S2: Epistatic filtration and parallel epistatic units for transport from $* * * * 0$ to $* * * * 1$ within the Khan data.

(a)

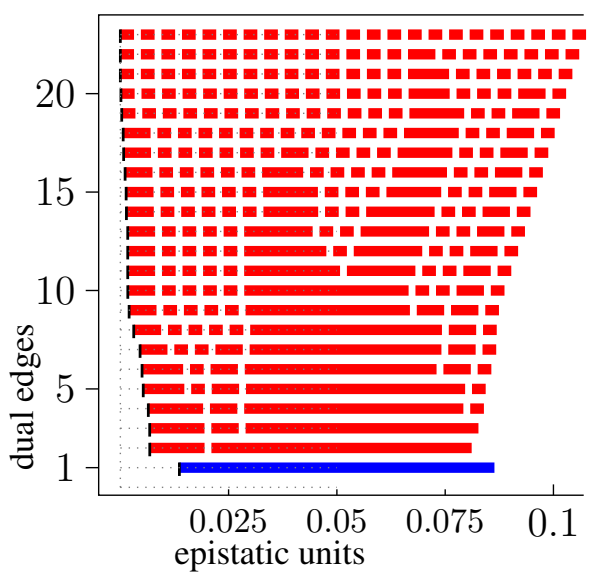

(b)

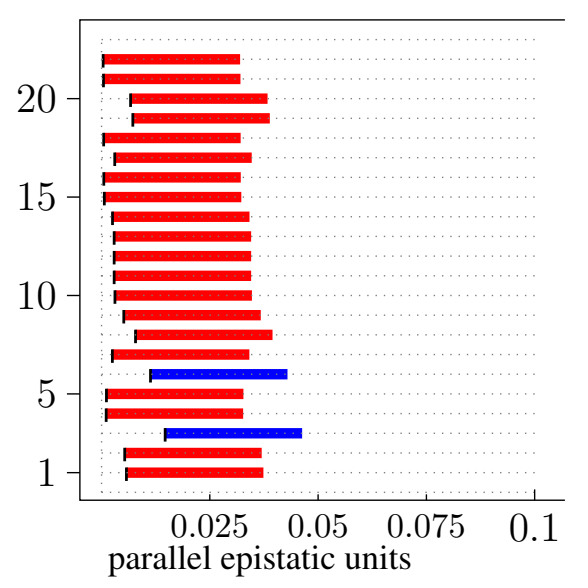

Figure S3: Epistatic filtration and parallel epistatic units for transport from $* * * * 1$ to $* * * * 0$ within the Khan data. 


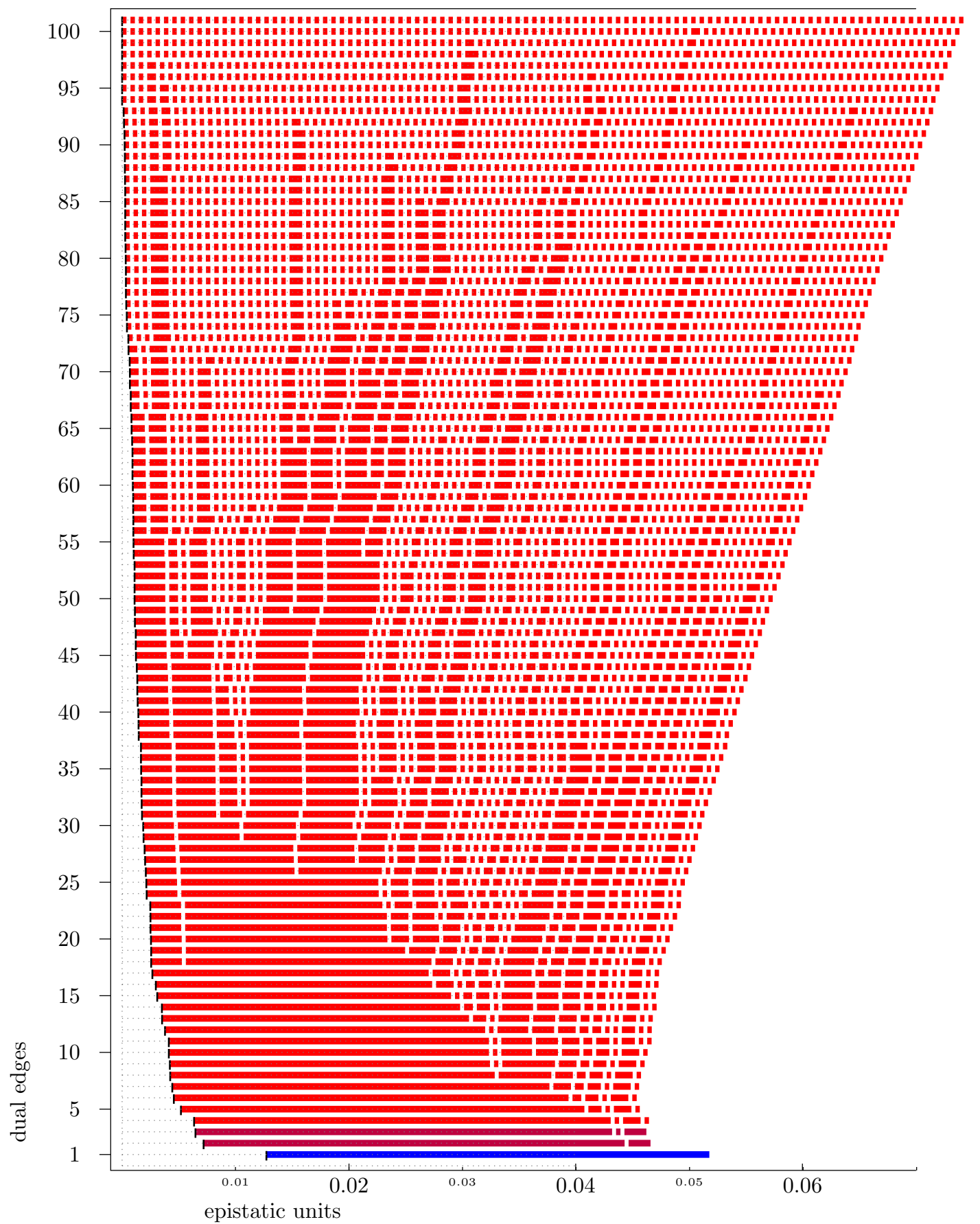

Figure S4: Complete filtration of the Khan data over the whole 5-cube. 

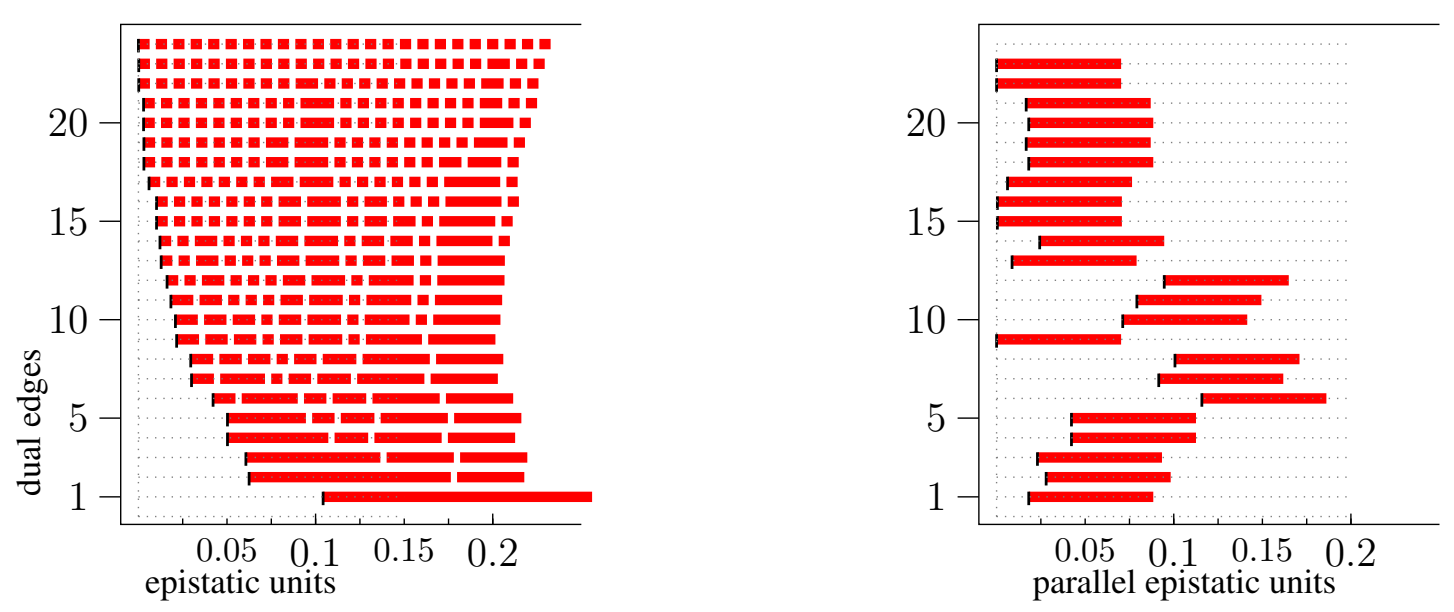

Figure S5: Parallel transport from $0 * * * *$ to $1 * * * *$ within the Tan data. Analysis based on mean values only; hence there is no color coding for the significance.
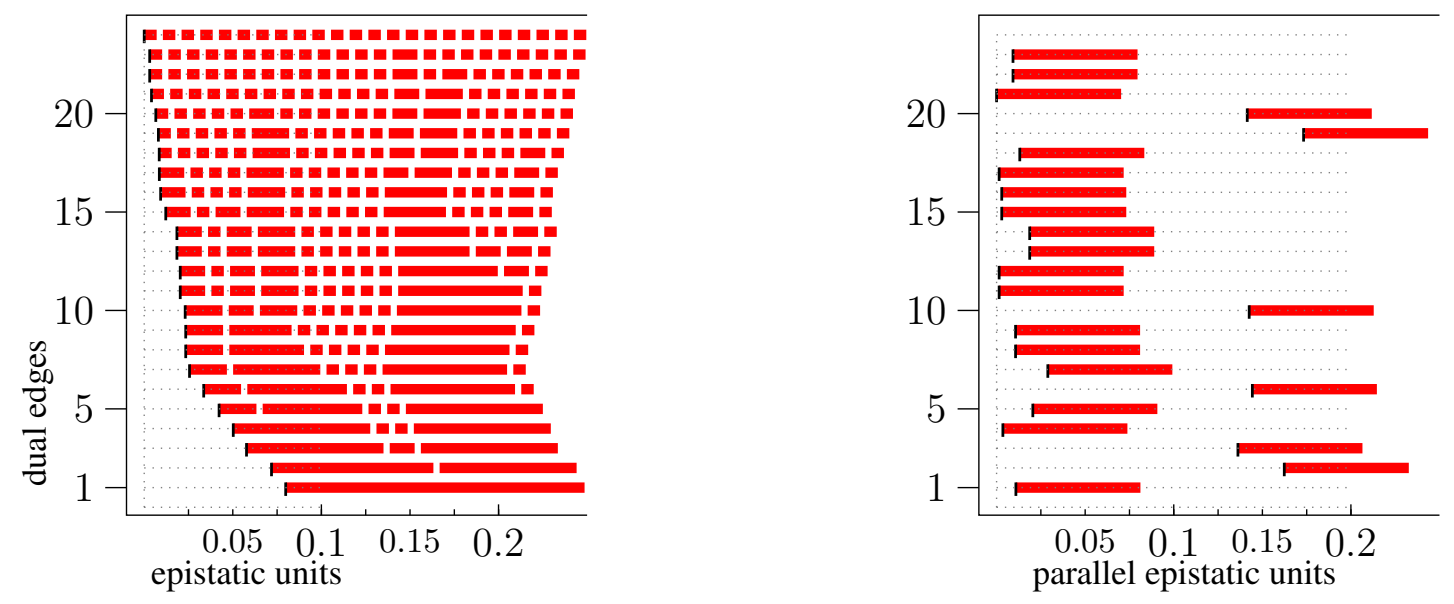

Figure S6: Parallel transport from the face $* * 0 * *$ to the face $* * 1 * *$ within the Tan data. Analysis based on mean values only; hence there is no color coding for the significance. 

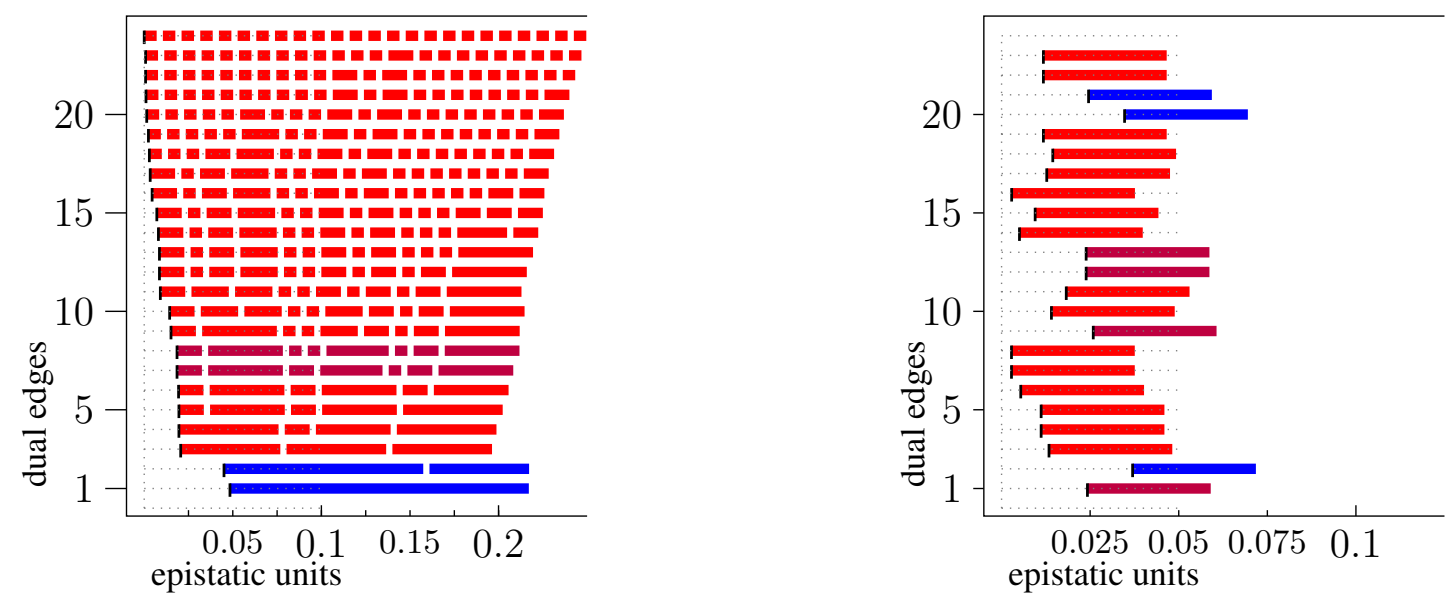

Figure S7: $0 * * * *($ Eble $)$ to $1 * * * *($ Eble $)$.
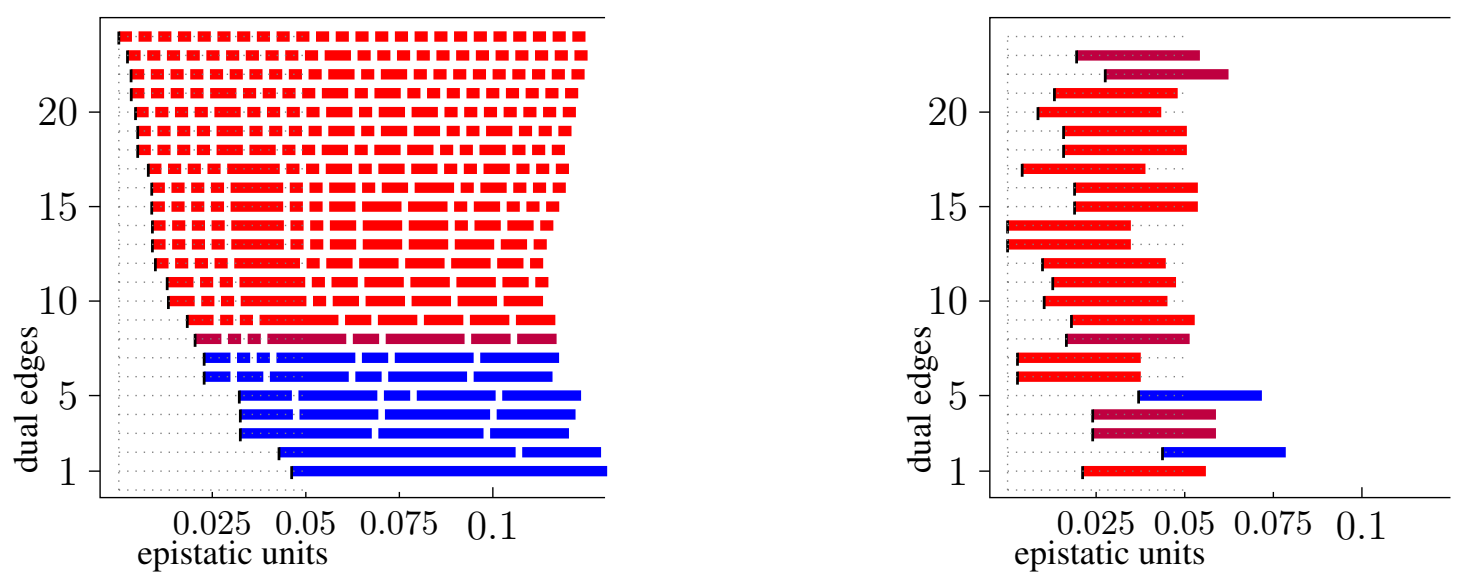

Figure S8: $* 0 * * *($ Eble $)$ to $* 1 * * *($ Eble $)$.
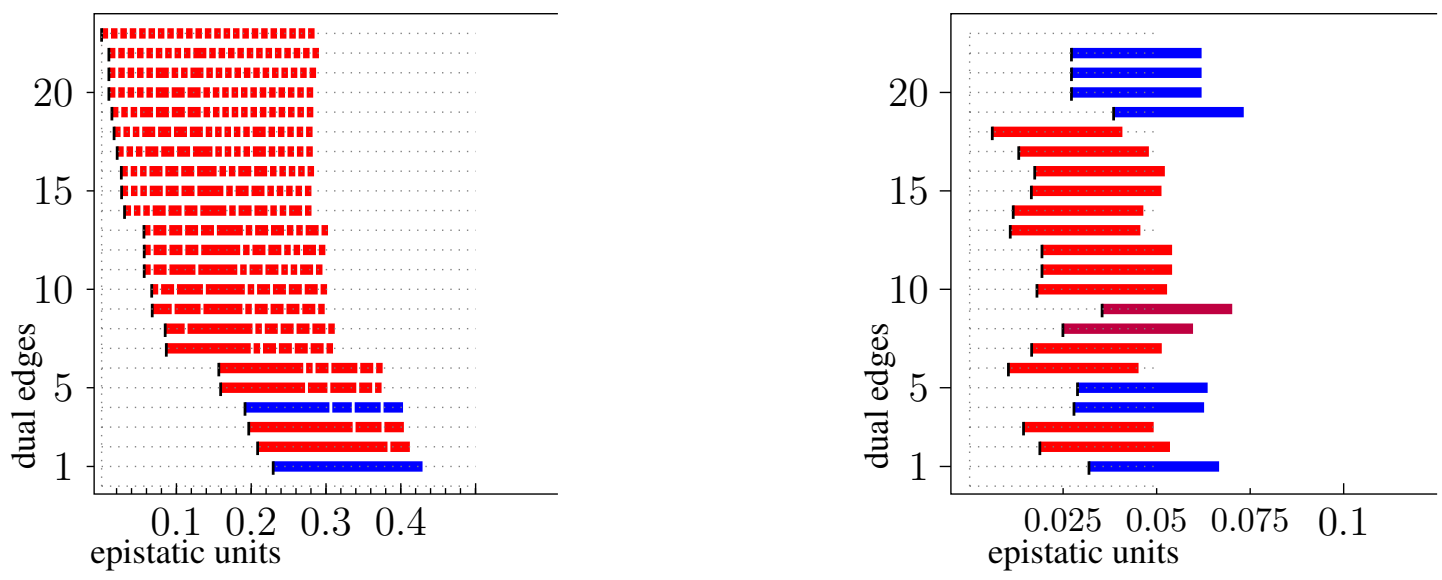

Figure S9: $0 * * * *($ GouldCFU) to $0 * * * *($ GouldTTD). 

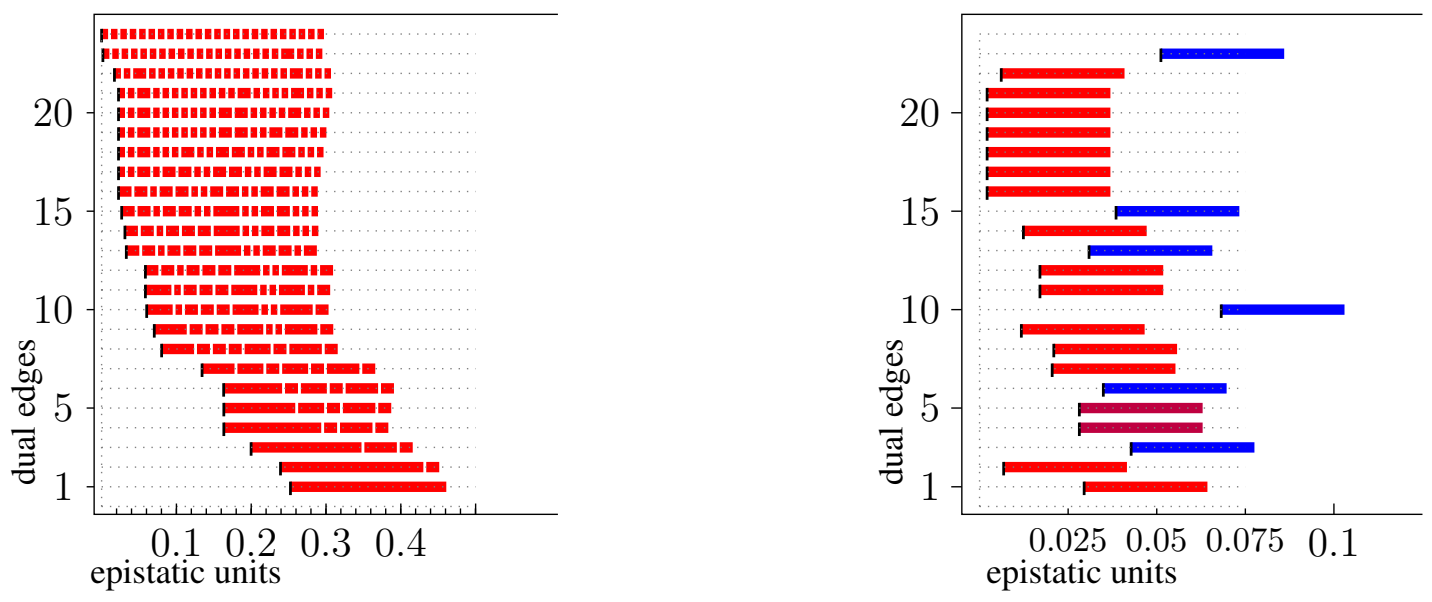

Figure S10: $1 * * * *($ GouldCFU) to $1 * * * *($ GouldTTD).
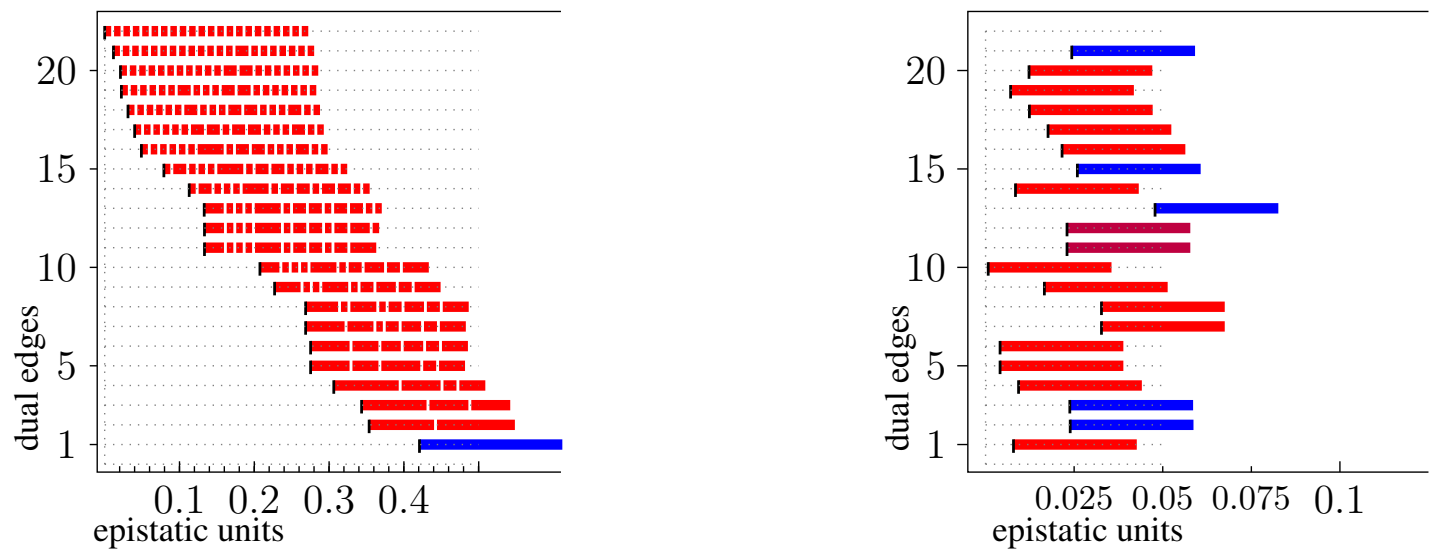

Figure S11: $* 0 * * *($ GouldCFU) to $* 0 * * *($ GouldTTD $)$.
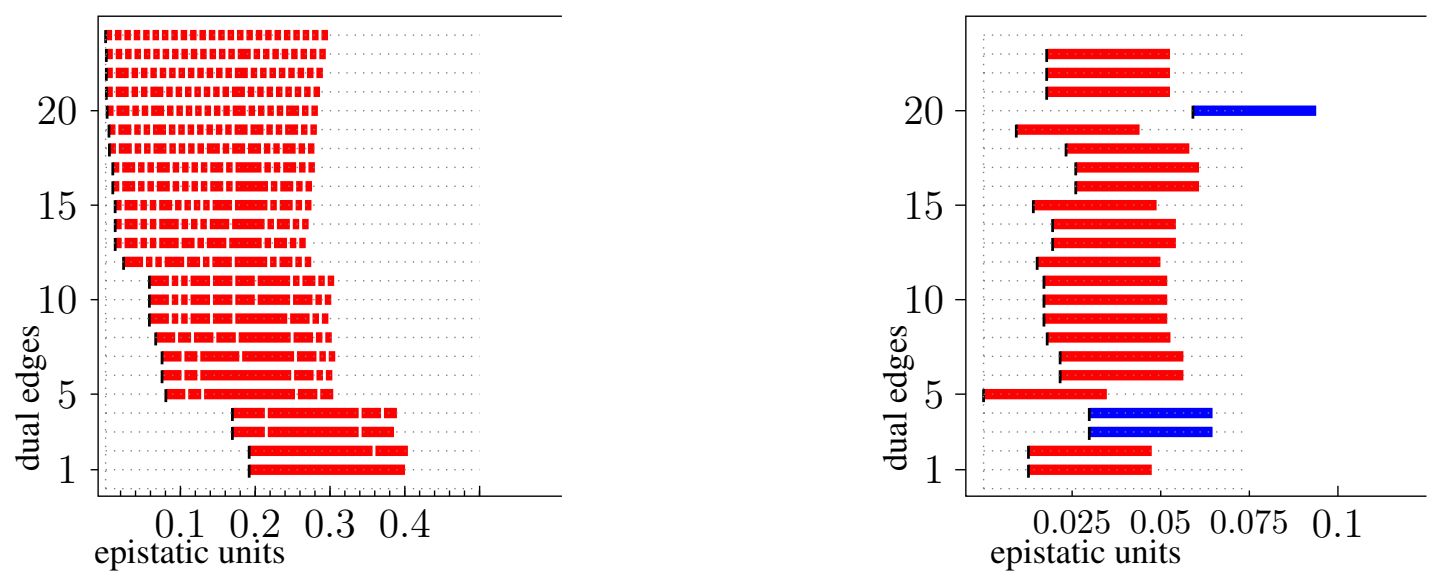

Figure S12: $* 1 * * *($ GouldCFU) to $* 1 * * *($ GouldTTD $)$. 
bioRxiv preprint doi: https://doi.org/10.1101/2021.09.11.459926; this version posted September 12, 2021. The copyright holder for this preprint (which was not certified by peer review) is the author/funder. All rights reserved. No reuse allowed without permission.

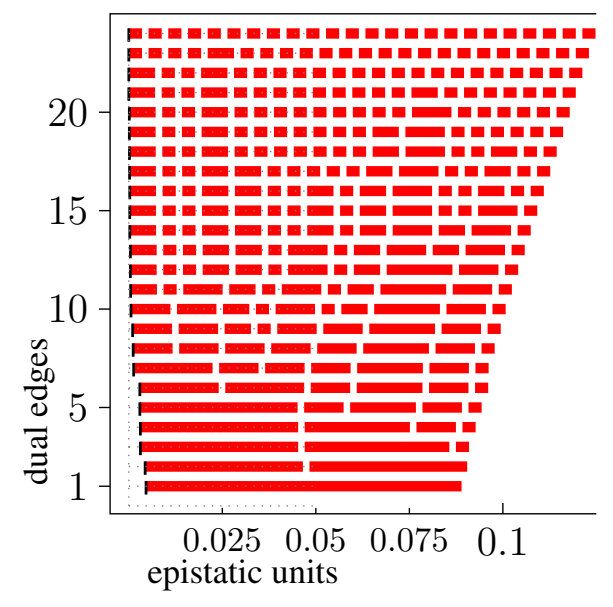

Figure S13: Product model associated to the parallel transport $0 * * 0 * \rightarrow 1 * * 0 *$ within the Khan evolution data, cf. (Fig. S1.) 
bioRxiv preprint doi: https://doi.org/10.1101/2021.09.11.459926; this version posted September 12, 2021. The copyright holder for this preprint (which was not certified by peer review) is the author/funder. All rights reserved. No reuse allowed without permission.

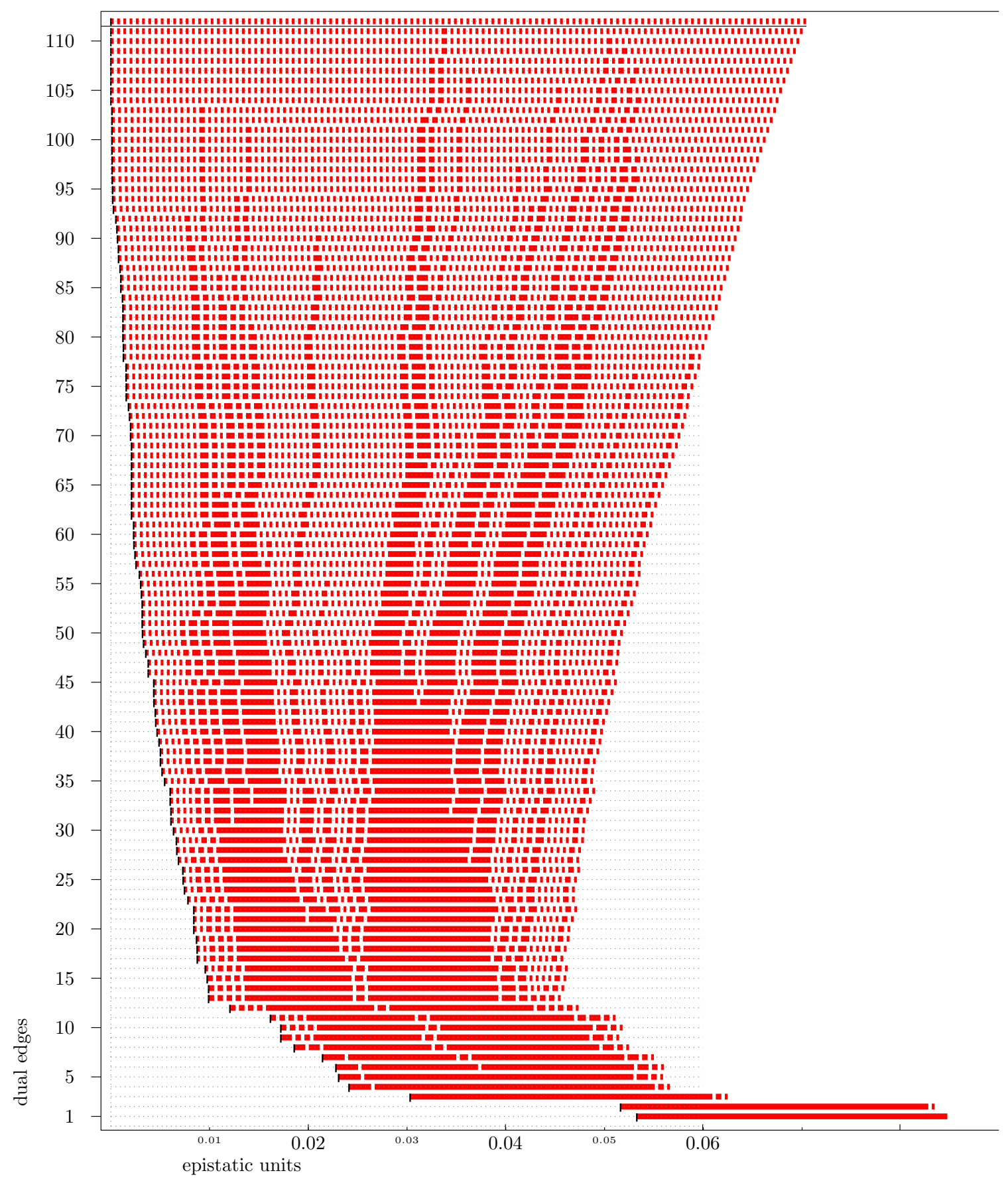

Figure S14: Non-generic product model associated to the parallel transport $* * 0 * * \rightarrow * * 1 * *$ within the Tan data. Its unique non-simplicial maximal cell has 7 vertices and is split into a bipyramid by a slight perturbation of its height values, cf. Theorem 8 of (38). The corresponding artificial dual edge has edge label 111 and is indicated by a horizontal line. 
bioRxiv preprint doi: https://doi.org/10.1101/2021.09.11.459926; this version posted September 12, 2021. The copyright holder for this preprint (which was not certified by peer review) is the author/funder. All rights reserved. No reuse allowed without permission.

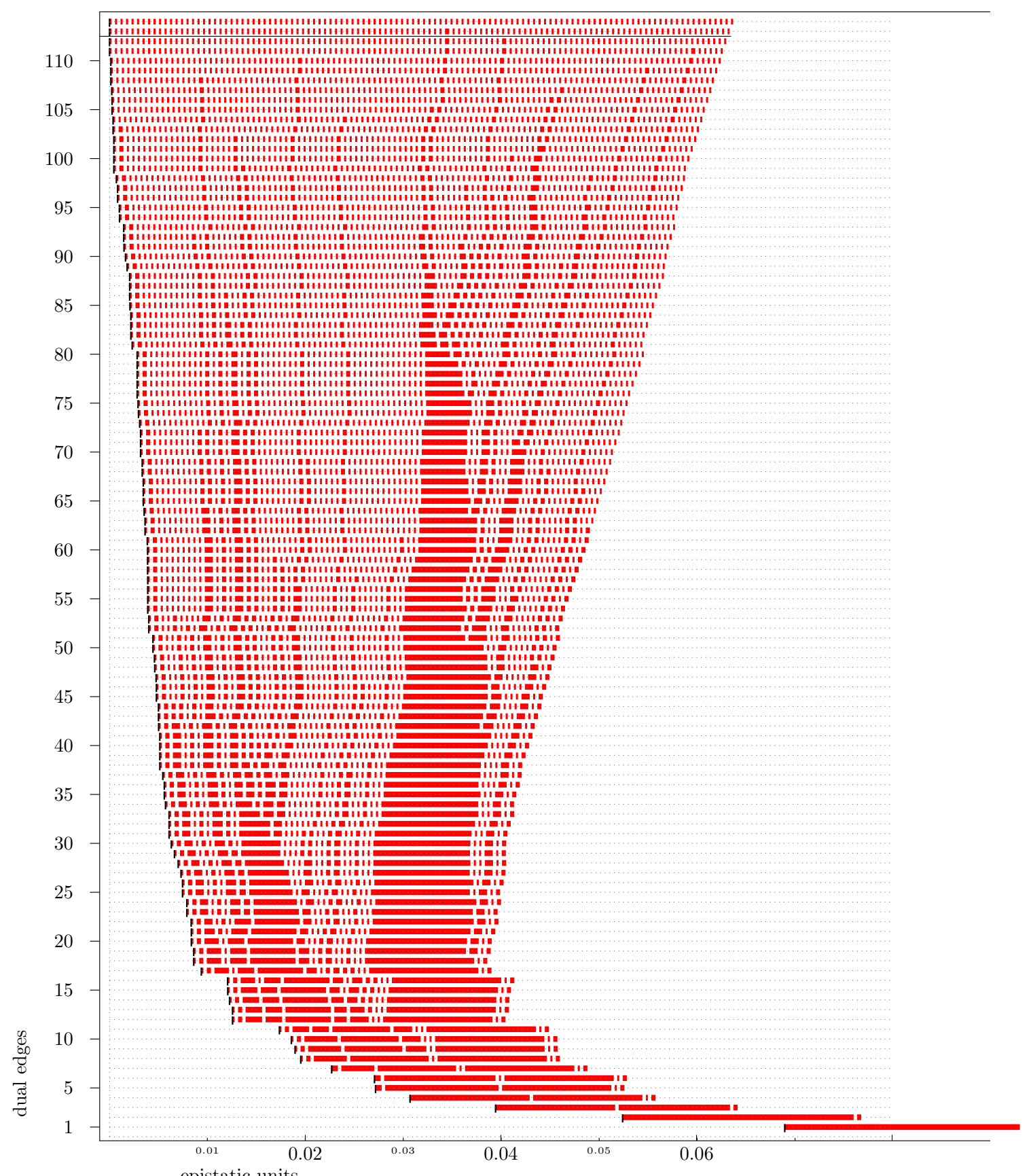

Figure S15: Non-generic product model associated to the parallel transport $0 * * * * \rightarrow 1 * * * *$ within the Tan data. There are two non-simplicial maximal cells, both of cardinality 7. As in (Fig. S14) they are split into a bipyramid each at the beginning of the filtration process. 


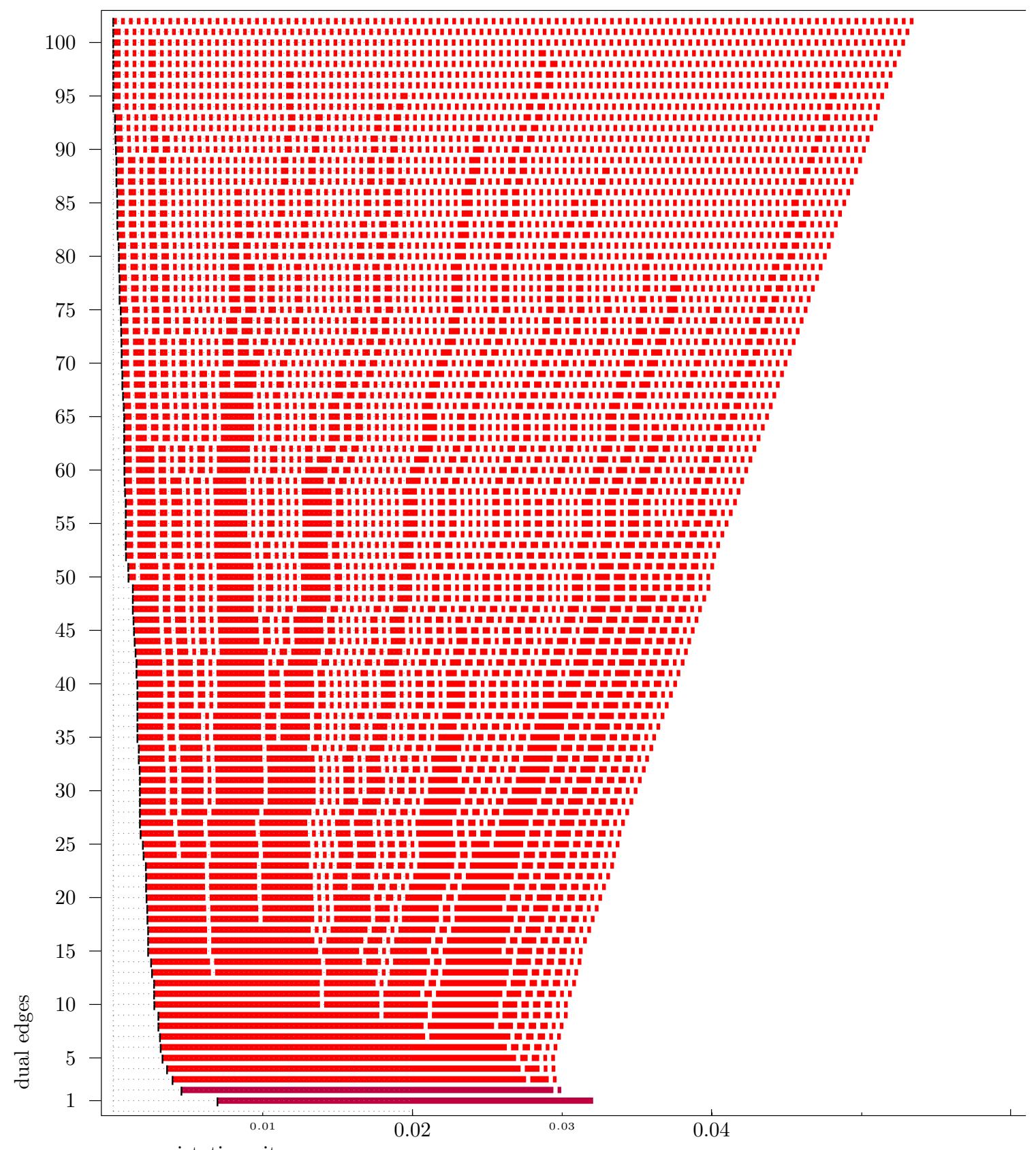

epistatic units

Figure S16: Product model for the parallel transport Khan $* * * 0 * \rightarrow * * * 1 *$. The semisignificant bipyramid labeled 2 reads $\left\{(1000)_{o}\right\}+\left\{(0000)_{o},(1010)_{0},(0110)_{o},(1010)_{p},(0011)_{p}\right\}+\left\{(0010)_{o}\right\}$ and the semisignificant bipyramid labeled 1 reads $\left\{(1100)_{o}\right\}+\left\{(1011)_{o},(1010)_{p},(1001)_{p},(0011)_{p},(1111)_{p}\right\}+\left\{(1011)_{p}\right\}$. 


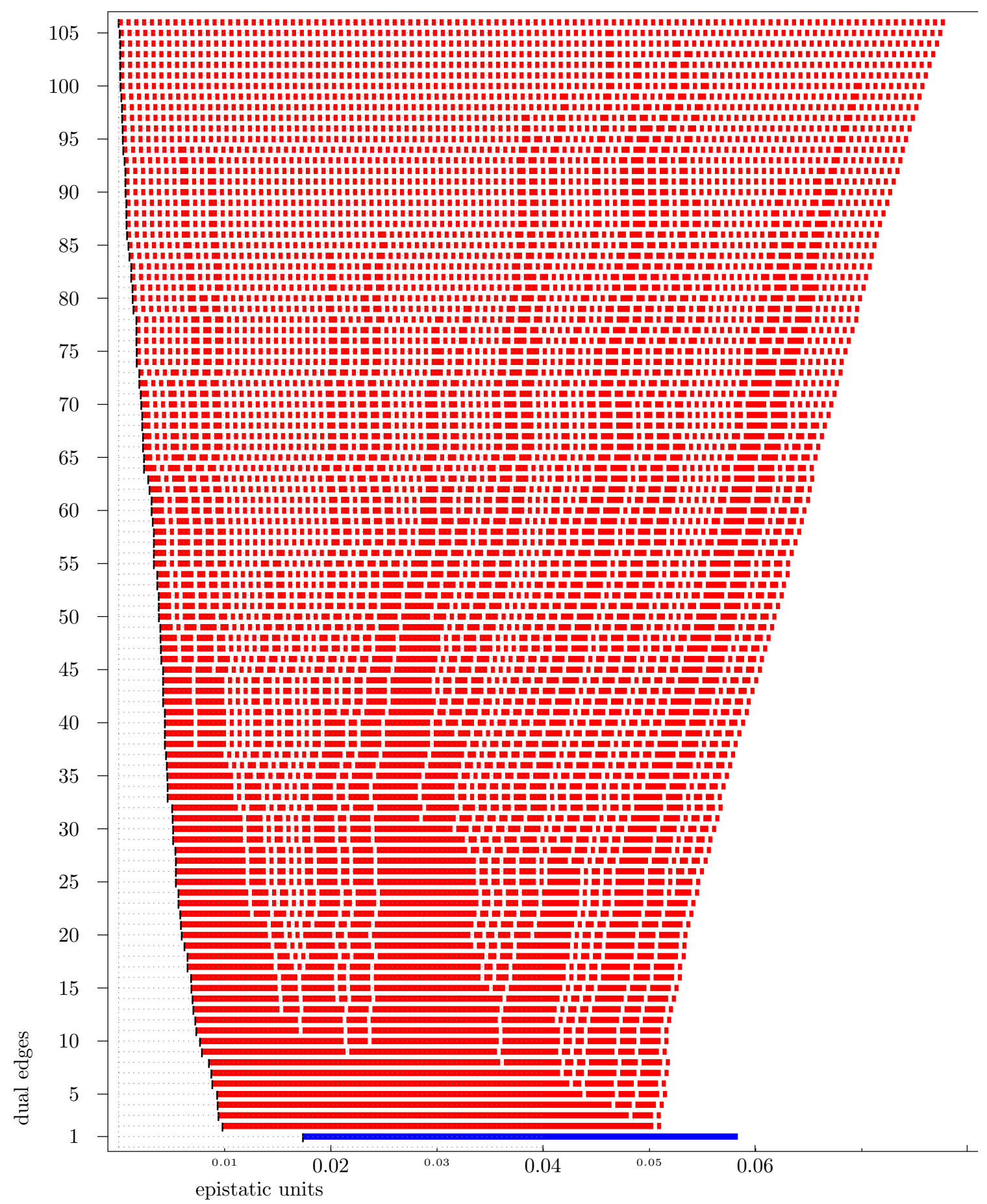

Figure S17: Product model for the parallel transport Eble $0 * * * * \rightarrow 1 * * * *$. The unique significant bipyramid reads $\left\{(0001)_{o}\right\}+\left\{(0000)_{o},(1001)_{o},(0101)_{o},(0011)_{o},(0001)_{p}\right\}+\left\{(0101)_{p}\right\}$ 


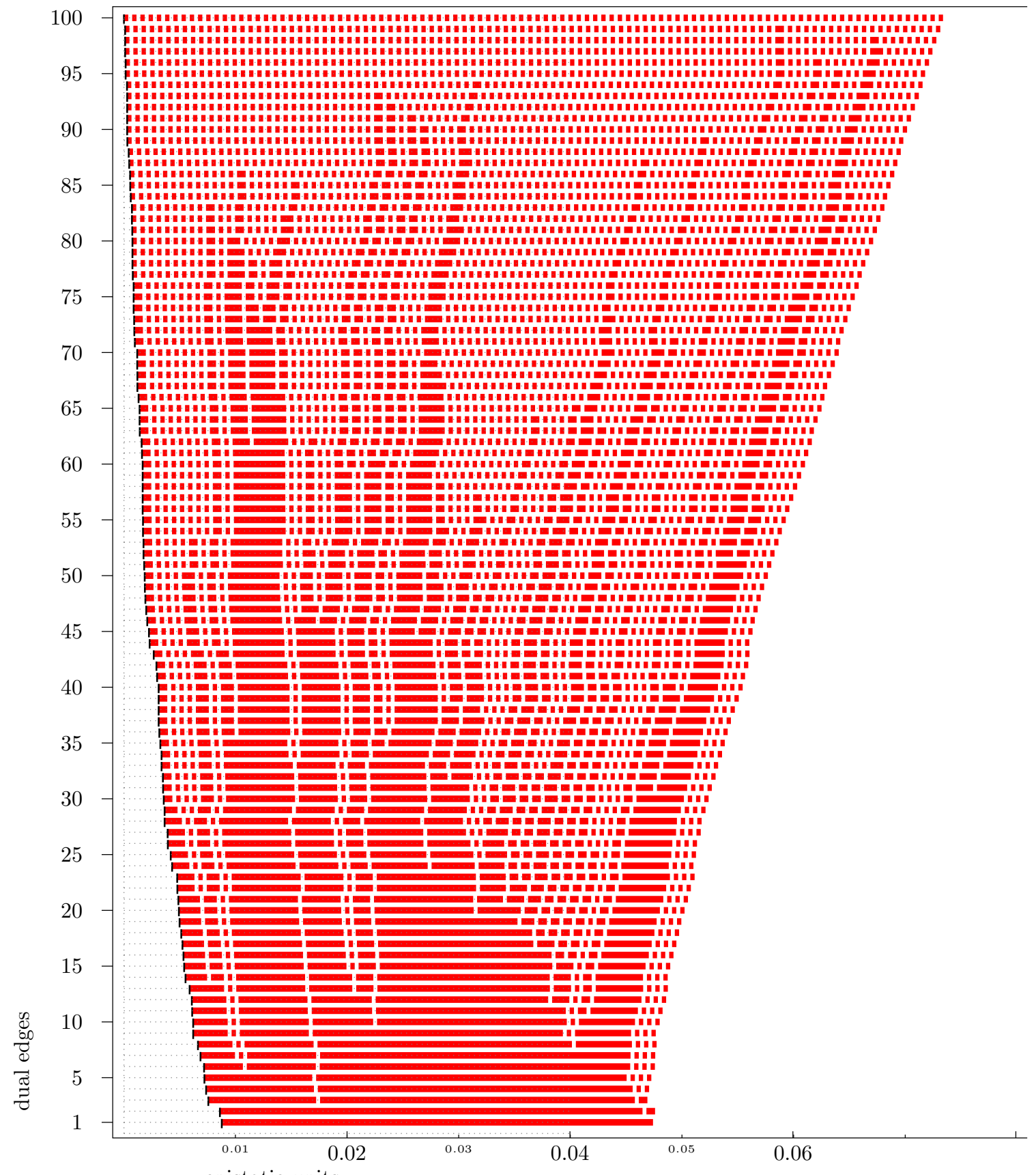

epistatic units

Figure S18: Product model for the parallel transport Eble $* 0 * * * \rightarrow * 1 * * *$. 
(a)

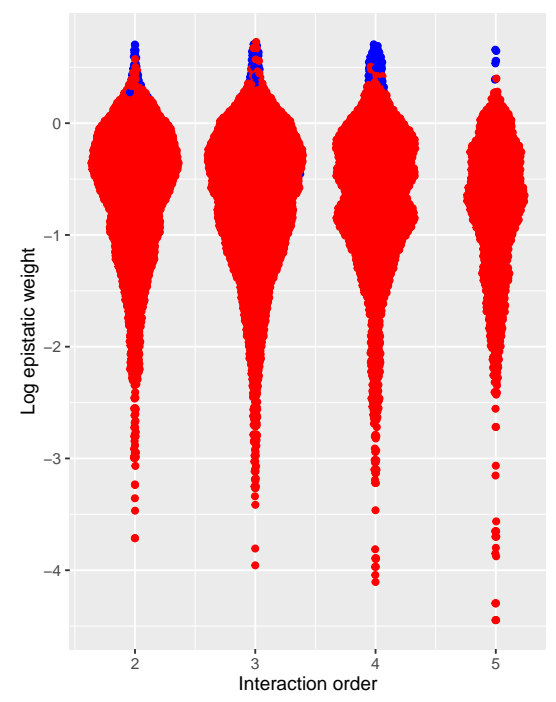

(b)

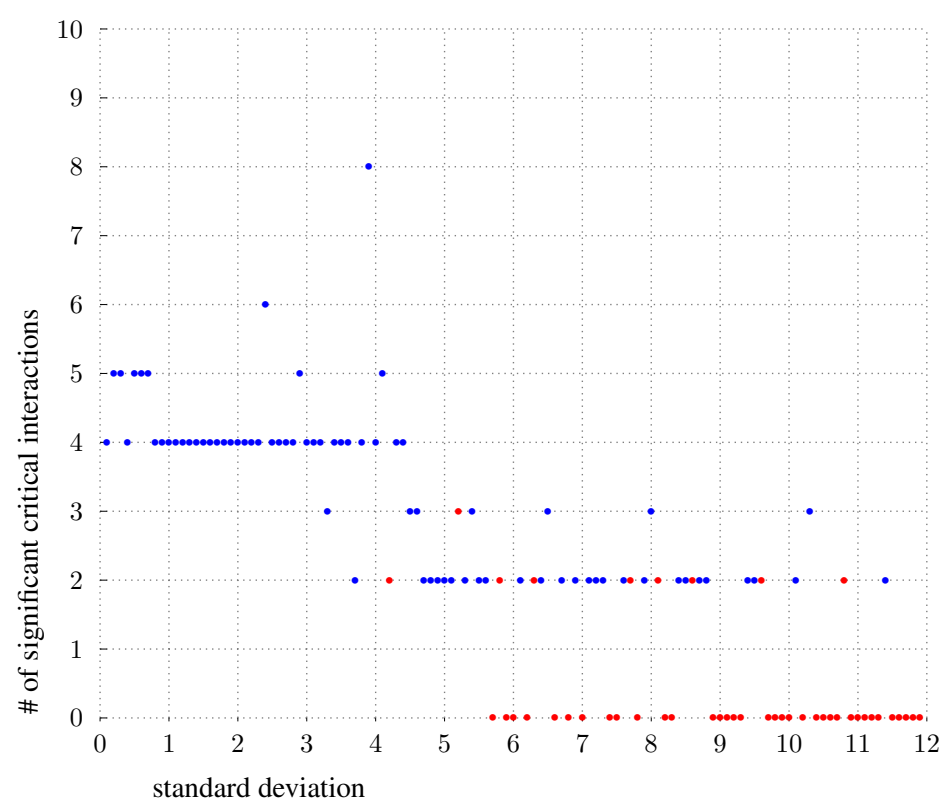

Figure S19: Synthetic data demonstrate method performance. Synthetic height functions over the 4dimensional cube are generated with 100 replicates each and standard deviation as indicated. The heights of the wild type 0000 and 0001 are sampled with mean 53, all the other vertices with mean 50 . (a) The distribution of $\log _{10}$-transformed epistatic weights is roughly constant as a function of interaction order, indicating the dimensional normalization is effective. (b) The number of significant interactions decreases as the standard deviation of the input data for each genotype increases. A blue dot is drawn if the interaction is significant and a red dot is drawn otherwise. 


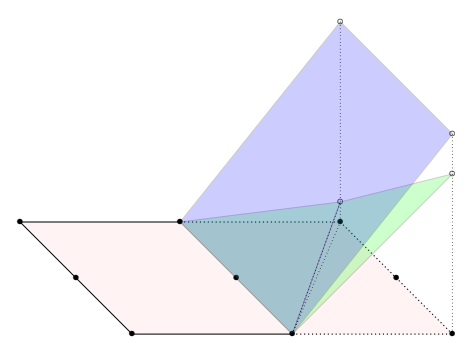

(a)

(b)

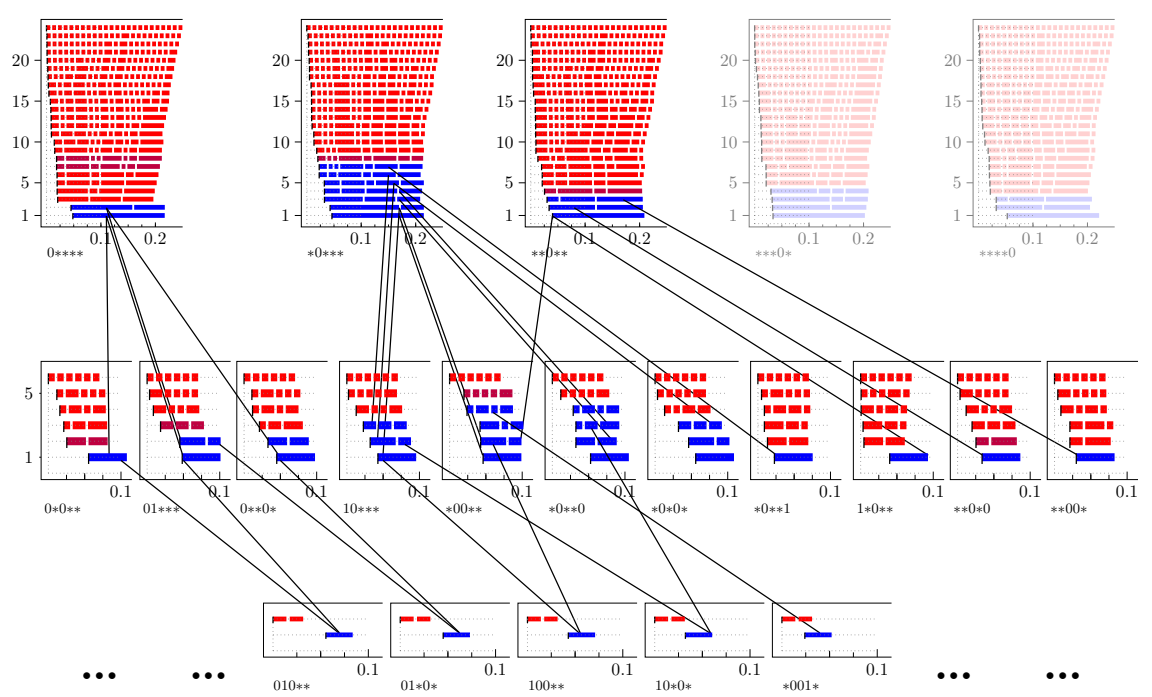

(c)
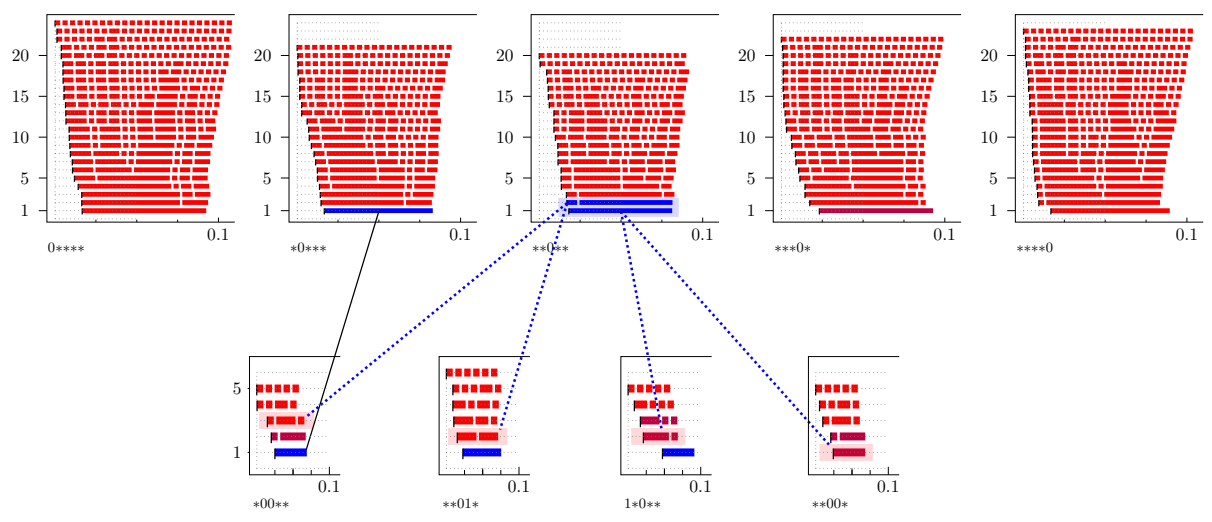

Figure S20: Meta-epistatic charts illustrate whether or not higher-order interactions arise from lower-order interactions. (a) Cartoon of the principle underlying meta-epistatic charts. The important loci in the interaction are depicted as black dots in a hyperplane through the genotypes, where the true dimensions of the genotypes are flattened onto the cartoon plane (pink). Higher-order interactions that derive from lower-order interactions occur in a new hyperplane (blue), which magnifies the weights of a subset of the landscape. In contrast, novel higher-order interactions that only arise in higher dimensions do not lie in a single additional hyperplane but instead require at least two additional hyperplanes (green). In (b) and (c) two meta-epistatic charts are represented. In each chart we identify the source of a higher-order interaction for the Eble and Gould data respectively. The results are compiled in Table S6. 
bioRxiv preprint doi: https://doi.org/10.1101/2021.09.11.459926; this version posted September 12, 2021. The copyright holder for this preprint (which was not certified by peer review) is the author/funder. All rights reserved. No reuse allowed without permission.

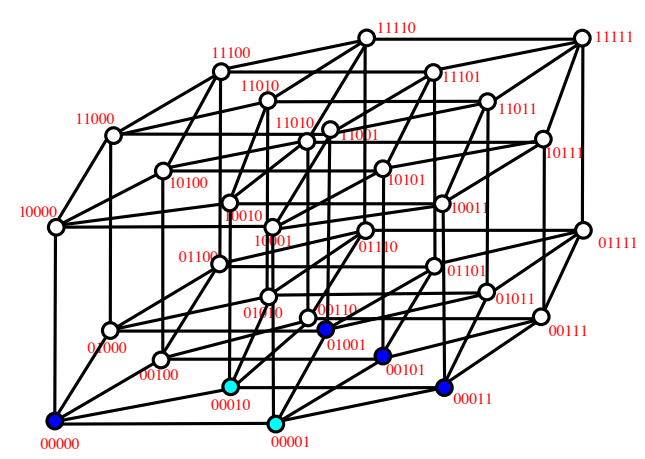

Figure S21: Vertices of the bipyramid $\{00001\}+\{00000,01001,00101,00011\}+\{00010\}$ arising for the Khan data set (37) restricted to $n=4$ loci. Dark blue dots correspond to common face $s \cap t$ of the bipyramid and light blue dots correspond to the satellite vertices of $s$ and $t$. 


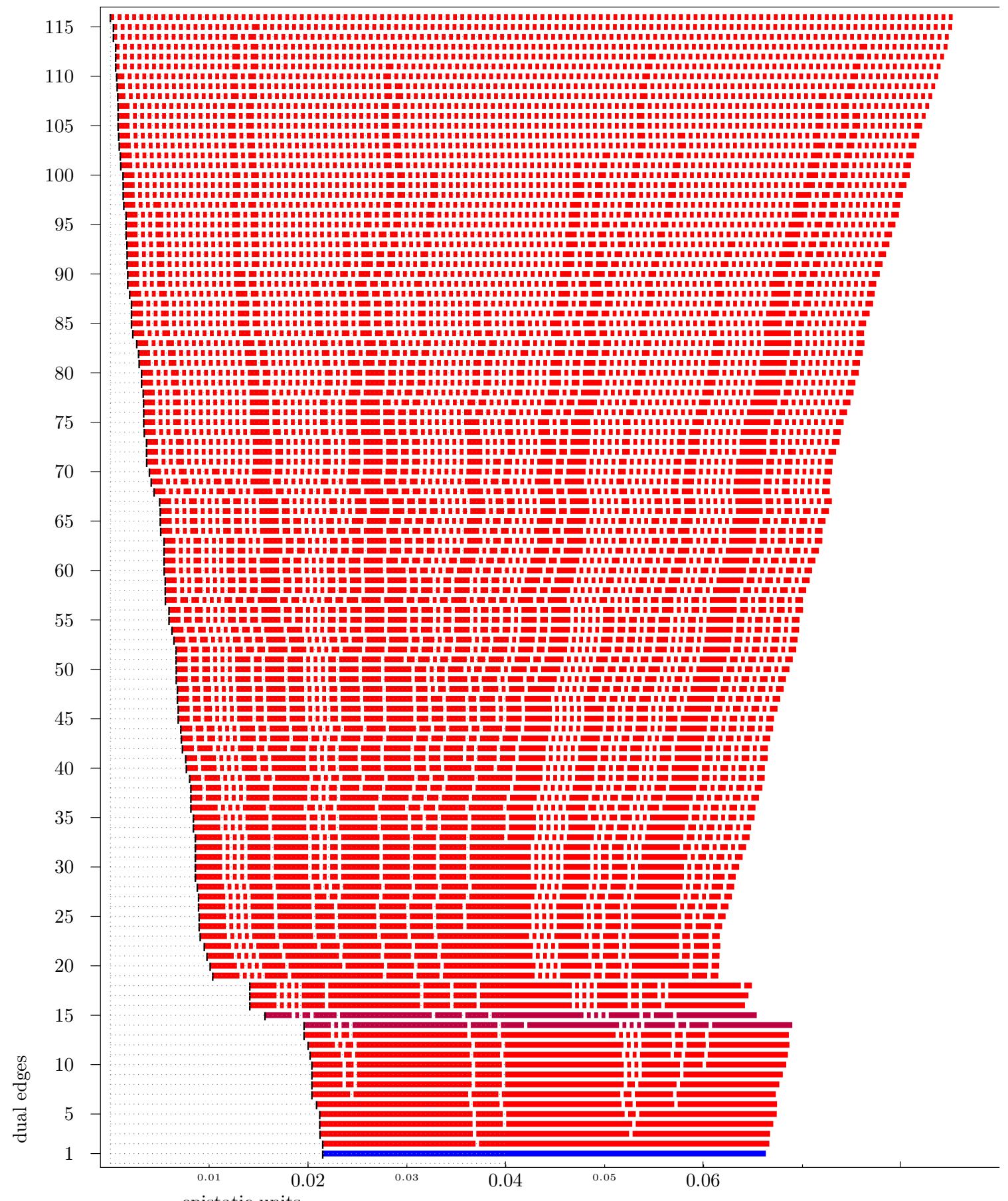

epistatic units

Figure S22: Complete filtration of the Eble fitness landscape over the whole 5-cube. 
Table S1: Number of circuits of $[0,1]^{n}$ and bipyramids among these.

\begin{tabular}{rrrr}
\hline dimensions & circuits & bipyramids & percentage \\
\hline 2 & 1 & 1 & $100.00 \%$ \\
3 & 20 & 8 & $40.00 \%$ \\
4 & 1348 & 1088 & $80.71 \%$ \\
5 & 353616 & 309056 & $87.40 \%$ \\
\hline
\end{tabular}


Table S2: Parallel analysis GouldCFU $0 * * * * \rightarrow$ Gould $0 * * * *$, non-critical red/red-case omitted.

\begin{tabular}{|c|c|c|c|c|c|c|c|c|}
\hline No. & bipyramid & type & $e_{o}$ & $e_{p}$ & $e_{o} / e_{p}$ & $p_{o}$ & $p_{p}$ & $p_{o} / p_{p}$ \\
\hline 22 & $\{01001\}+\{01000,01100,01010,00111\}+\{00110\}$ & red/blue & 0.010 & 0.027 & 0.357 & 0.978 & 0.038 & 25.873 \\
\hline 21 & $\{01001\}+\{01000,00100,01100,00111\}+\{00110\}$ & red/blue & 0.010 & 0.027 & 0.357 & 0.978 & 0.038 & 25.873 \\
\hline 20 & $\{01001\}+\{01000,00010,01010,00111\}+\{00110\}$ & red/blue & 0.010 & 0.027 & 0.357 & 0.978 & 0.038 & 25.873 \\
\hline 19 & $\{01001\}+\{01000,00100,00010,00111\}+\{00110\}$ & red/blue & 0.014 & 0.039 & 0.357 & 0.978 & 0.038 & 25.873 \\
\hline 18 & $\{01100\}+\{01001,01110,01101,00111\}+\{01111\}$ & $\mathrm{red} / \mathrm{red}$ & 0.017 & 0.006 & 2.747 & 0.815 & 0.677 & 1.204 \\
\hline 17 & $\{01000\}+\{01100,01010,00110,00111\}+\{01110\}$ & $\mathrm{red} / \mathrm{red}$ & 0.021 & 0.013 & 1.584 & 0.783 & 0.433 & 1.808 \\
\hline 16 & $\{00100\}+\{01100,01001,00101,00111\}+\{01101\}$ & $\mathrm{red} / \mathrm{red}$ & 0.026 & 0.017 & 1.514 & 0.807 & 0.302 & 2.672 \\
\hline 15 & $\{01001\}+\{01100,01010,01110,00111\}+\{00110\}$ & $\mathrm{red} / \mathrm{red}$ & 0.027 & 0.017 & 1.619 & 0.941 & 0.231 & 4.074 \\
\hline 14 & $\{00001\}+\{00010,01001,00011,00111\}+\{01011\}$ & $\mathrm{red} / \mathrm{red}$ & 0.031 & 0.012 & 2.630 & 0.905 & 0.312 & 2.901 \\
\hline 13 & $\{01000\}+\{00100,00010,00001,01001\}+\{00111\}$ & $\mathrm{red} / \mathrm{red}$ & 0.057 & 0.011 & 5.217 & 0.869 & 0.479 & 1.814 \\
\hline 12 & $\{00010\}+\{01000,01010,00110,00111\}+\{01100\}$ & $\mathrm{red} / \mathrm{red}$ & 0.057 & 0.019 & 2.943 & 0.531 & 0.148 & 3.588 \\
\hline \multirow[t]{3}{*}{11} & $\{00010\}+\{01000,00100,00110,00111\}+\{01100\}$ & $\mathrm{red} / \mathrm{red}$ & 0.057 & 0.019 & 2.943 & 0.531 & 0.148 & 3.588 \\
\hline & $\{00010\}+\{01000,01010,01001,00111\}+\{01100\}$ & red/blue & 0.067 & 0.047 & 1.431 & 0.853 & 0.032 & 27.079 \\
\hline & $\{00010\}+\{01000,00100,01001,00111\}+\{01100\}$ & red/blue & 0.067 & 0.047 & 1.431 & 0.853 & 0.032 & 27.079 \\
\hline 10 & $\{01010\}+\{01001,01110,01011,00111\}+\{01111\}$ & $\mathrm{red} / \mathrm{red}$ & 0.067 & 0.018 & 3.722 & 0.323 & 0.186 & 1.737 \\
\hline 9 & $\{00000\}+\{01000,00100,00010,00001\}+\{01001\}$ & $\mathrm{red} / \mathrm{red}$ & 0.068 & 0.035 & 1.911 & 0.851 & 0.086 & 9.872 \\
\hline 8 & $\{00100\}+\{01000,01100,00110,00111\}+\{01010\}$ & $\mathrm{red} / \mathrm{red}$ & 0.085 & 0.025 & 3.408 & 0.317 & 0.083 & 3.819 \\
\hline \multirow[t]{3}{*}{7} & $\{01000\}+\{00100,00010,01001,00111\}+\{00001\}$ & $\mathrm{red} / \mathrm{red}$ & 0.087 & 0.017 & 5.217 & 0.869 & 0.479 & 1.814 \\
\hline & $\{00100\}+\{01000,01100,01001,00111\}+\{01010\}$ & red/blue & 0.095 & 0.052 & 1.816 & 0.791 & 0.019 & 40.984 \\
\hline & $\{00100\}+\{01000,00010,01001,00111\}+\{01010\}$ & red/blue & 0.095 & 0.052 & 1.816 & 0.791 & 0.019 & 40.984 \\
\hline 6 & $\{01101\}+\{01001,01110,00111,01111\}+\{01011\}$ & $\mathrm{red} / \mathrm{red}$ & 0.157 & 0.010 & 15.097 & 0.533 & 0.362 & 1.472 \\
\hline 5 & $\{00001\}+\{00100,01001,00101,00111\}+\{01100\}$ & red/blue & 0.159 & 0.029 & 5.516 & 0.541 & 0.028 & 19.049 \\
\hline 4 & $\{01010\}+\{00010,01001,01011,00111\}+\{00011\}$ & blue/blue & 0.192 & 0.028 & 6.871 & 0.032 & 0.042 & 0.758 \\
\hline 3 & $\{00010\}+\{00100,00001,01001,00111\}+\{00101\}$ & $\mathrm{red} / \mathrm{red}$ & 0.197 & 0.014 & 13.654 & 0.262 & 0.211 & 1.242 \\
\hline 2 & $\{01100\}+\{01010,01001,01110,00111\}+\{01011\}$ & $\mathrm{red} / \mathrm{red}$ & 0.209 & 0.019 & 11.109 & 0.502 & 0.175 & 2.869 \\
\hline \multirow[t]{2}{*}{1} & $\{01000\}+\{00010,01010,01001,00111\}+\{01011\}$ & blue/blue & 0.229 & 0.032 & 7.188 & 0.458 & 0.049 & 9.271 \\
\hline & $\{00100\}+\{00010,00001,01001,00111\}+\{00011\}$ & blue/red & 0.365 & 0.007 & 53.243 & 0.026 & 0.526 & 0.049 \\
\hline
\end{tabular}


Table S3: Parallel analysis GouldCFU $1 * * * * \rightarrow$ Gould 1****, non-critical red/red-case omitted.

\begin{tabular}{|c|c|c|c|c|c|c|c|c|}
\hline No. & bipyramid & type & $e_{o}$ & $e_{p}$ & $e_{o} / e_{p}$ & $p_{o}$ & $p_{p}$ & $p_{o} / p_{p}$ \\
\hline 23 & $\{11001\}+\{11000,10101,11101,11011\}+\{11111\}$ & $\mathrm{red} / \mathrm{blue}$ & 0.002 & 0.051 & 0.033 & 0.962 & 0.001 & 1286.096 \\
\hline 22 & $\{11100\}+\{11000,10101,11110,11101\}+\{11111\}$ & $\mathrm{red} / \mathrm{red}$ & 0.017 & 0.006 & 2.799 & 0.773 & 0.689 & 1.122 \\
\hline 21 & $\{10000\}+\{11010,10101,10011,10111\}+\{11111\}$ & $\mathrm{red} / \mathrm{red}$ & 0.023 & 0.002 & 10.615 & 0.967 & 0.875 & 1.105 \\
\hline 20 & $\{10000\}+\{11010,10101,10011,11011\}+\{11111\}$ & $\mathrm{red} / \mathrm{red}$ & 0.023 & 0.002 & 10.615 & 0.967 & 0.875 & 1.105 \\
\hline 19 & $\{10000\}+\{11000,11010,10101,11011\}+\{11111\}$ & $\mathrm{red} / \mathrm{red}$ & 0.023 & 0.002 & 10.615 & 0.967 & 0.875 & 1.105 \\
\hline 18 & $\{10000\}+\{11010,10110,10101,10111\}+\{11111\}$ & $\mathrm{red} / \mathrm{red}$ & 0.023 & 0.002 & 10.615 & 0.967 & 0.875 & 1.105 \\
\hline 17 & $\{10000\}+\{11010,10110,10101,11110\}+\{11111\}$ & $\mathrm{red} / \mathrm{red}$ & 0.023 & 0.002 & 10.615 & 0.967 & 0.875 & 1.105 \\
\hline 16 & $\{10000\}+\{11000,11010,10101,11110\}+\{11111\}$ & $\mathrm{red} / \mathrm{red}$ & 0.023 & 0.002 & 10.615 & 0.967 & 0.875 & 1.105 \\
\hline 15 & $\{11011\}+\{11010,10101,10011,11111\}+\{10111\}$ & $\mathrm{red} / \mathrm{blue}$ & 0.027 & 0.039 & 0.695 & 0.580 & 0.012 & 47.154 \\
\hline 14 & $\{10110\}+\{10000,10010,11010,10111\}+\{10011\}$ & $\mathrm{red} / \mathrm{red}$ & 0.031 & 0.012 & 2.513 & 0.693 & 0.277 & 2.502 \\
\hline 13 & $\{11001\}+\{10000,10001,10101,11011\}+\{10011\}$ & $\mathrm{red} / \mathrm{blue}$ & 0.033 & 0.031 & 1.066 & 0.388 & 0.007 & 54.190 \\
\hline 12 & $\{11010\}+\{11000,10101,11011,11111\}+\{11101\}$ & $\mathrm{red} / \mathrm{red}$ & 0.059 & 0.017 & 3.428 & 0.905 & 0.318 & 2.846 \\
\hline 11 & $\{11010\}+\{11000,10101,11110,11111\}+\{11101\}$ & $\mathrm{red} / \mathrm{red}$ & 0.059 & 0.017 & 3.428 & 0.905 & 0.318 & 2.846 \\
\hline 10 & $\{11010\}+\{10000,11000,10101,11011\}+\{11001\}$ & $\mathrm{red} / \mathrm{blue}$ & 0.060 & 0.068 & 0.881 & 0.902 & 0.000 & $\infty$ \\
\hline 9 & $\{10000\}+\{11000,11100,10101,11110\}+\{11101\}$ & $\mathrm{red} / \mathrm{red}$ & 0.070 & 0.012 & 5.959 & 0.897 & 0.426 & 2.106 \\
\hline 8 & $\{10100\}+\{10000,11100,10110,10101\}+\{11110\}$ & $\mathrm{red} / \mathrm{red}$ & 0.080 & 0.021 & 3.820 & 0.430 & 0.274 & 1.569 \\
\hline 7 & $\{11110\}+\{11010,10110,10101,11111\}+\{10111\}$ & $\mathrm{red} / \mathrm{red}$ & 0.134 & 0.021 & 6.534 & 0.130 & 0.227 & 0.573 \\
\hline 6 & $\{11000\}+\{10000,11010,10101,11110\}+\{10110\}$ & $\mathrm{red} / \mathrm{blue}$ & 0.163 & 0.035 & 4.659 & 0.737 & 0.019 & 38.586 \\
\hline 5 & $\{10010\}+\{10000,11010,10110,10111\}+\{10101\}$ & $\mathrm{red} / \mathrm{red}$ & 0.163 & 0.028 & 5.788 & 0.776 & 0.075 & 10.402 \\
\hline \multirow[t]{2}{*}{4} & $\{10010\}+\{10000,11010,10011,10111\}+\{10101\}$ & $\mathrm{red} / \mathrm{red}$ & 0.163 & 0.028 & 5.788 & 0.776 & 0.075 & 10.402 \\
\hline & $\{11000\}+\{11010,10101,11110,11111\}+\{10110\}$ & red/blue & 0.186 & 0.037 & 5.000 & 0.695 & 0.026 & 26.834 \\
\hline 3 & $\{11000\}+\{10000,11100,10101,11110\}+\{10110\}$ & red/blue & 0.200 & 0.043 & 4.659 & 0.737 & 0.019 & 38.586 \\
\hline 2 & $\{11000\}+\{10000,11010,10101,11011\}+\{10011\}$ & $\mathrm{red} / \mathrm{red}$ & 0.239 & 0.007 & 35.102 & 0.621 & 0.628 & 0.989 \\
\hline \multirow[t]{3}{*}{1} & $\{11000\}+\{10000,11001,10101,11011\}+\{10001\}$ & $\mathrm{red} / \mathrm{red}$ & 0.253 & 0.030 & 8.530 & 0.671 & 0.104 & 6.452 \\
\hline & $\{11110\}+\{11000,11010,10101,11111\}+\{11011\}$ & $\mathrm{red} / \mathrm{blue}$ & 0.301 & 0.026 & 11.785 & 0.288 & 0.035 & 8.348 \\
\hline & $\{10001\}+\{10000,10101,10011,11011\}+\{11010\}$ & $\mathrm{red} / \mathrm{blue}$ & 0.313 & 0.039 & 8.062 & 0.598 & 0.014 & 43.650 \\
\hline
\end{tabular}


Table S4: Parallel analysis GouldCFU $* 0 * * * \rightarrow$ Gould $* 0 * * *$, non-critical red/red-case omitted.

\begin{tabular}{|c|c|c|c|c|c|c|c|c|}
\hline No. & bipyramid & type & $e_{o}$ & $e_{p}$ & $e_{o} / e_{p}$ & $p_{o}$ & $p_{p}$ & $p_{o} / p_{p}$ \\
\hline 21 & $\{10001\}+\{10000,00001,10101,10011\}+\{00111\}$ & $\mathrm{red} / \mathrm{blue}$ & 0.012 & 0.024 & 0.481 & 0.963 & 0.026 & 36.756 \\
\hline 20 & $\{00010\}+\{10000,10010,00011,00111\}+\{10011\}$ & $\mathrm{red} / \mathrm{red}$ & 0.021 & 0.012 & 1.714 & 0.797 & 0.270 & 2.952 \\
\hline 19 & $\{10100\}+\{10000,00100,10110,10101\}+\{00110\}$ & $\mathrm{red} / \mathrm{red}$ & 0.022 & 0.007 & 3.155 & 0.869 & 0.717 & 1.212 \\
\hline 18 & $\{10110\}+\{10000,10010,00111,10111\}+\{10011\}$ & $\mathrm{red} / \mathrm{red}$ & 0.031 & 0.012 & 2.513 & 0.693 & 0.277 & 2.502 \\
\hline 17 & $\{00100\}+\{10000,00110,10101,00111\}+\{10110\}$ & $\mathrm{red} / \mathrm{red}$ & 0.040 & 0.018 & 2.266 & 0.915 & 0.290 & 3.155 \\
\hline 16 & $\{00100\}+\{10000,00110,10110,10101\}+\{00111\}$ & $\mathrm{red} / \mathrm{red}$ & 0.049 & 0.022 & 2.266 & 0.915 & 0.290 & 3.155 \\
\hline 15 & $\{00001\}+\{10000,00100,00010,00111\}+\{00110\}$ & red/blue & 0.079 & 0.026 & 3.047 & 0.698 & 0.023 & 30.749 \\
\hline 14 & $\{00010\}+\{10000,10010,00110,00111\}+\{10110\}$ & $\mathrm{red} / \mathrm{red}$ & 0.113 & 0.008 & 13.352 & 0.295 & 0.461 & 0.640 \\
\hline 13 & $\{00000\}+\{10000,00100,00010,00001\}+\{00111\}$ & $\mathrm{red} / \mathrm{blue}$ & 0.133 & 0.048 & 2.775 & 0.476 & 0.001 & 707.281 \\
\hline 12 & $\{10010\}+\{10000,10011,00111,10111\}+\{10101\}$ & $\mathrm{red} / \mathrm{red}$ & 0.133 & 0.023 & 5.788 & 0.776 & 0.075 & 10.402 \\
\hline 11 & $\{10010\}+\{10000,10110,00111,10111\}+\{10101\}$ & $\mathrm{red} / \mathrm{red}$ & 0.133 & 0.023 & 5.788 & 0.776 & 0.075 & 10.402 \\
\hline 10 & $\{00011\}+\{10000,00001,10011,00111\}+\{10101\}$ & $\mathrm{red} / \mathrm{red}$ & 0.208 & 0.001 & 275.689 & 0.413 & 0.949 & 0.435 \\
\hline 9 & $\{00010\}+\{10000,00100,00001,00111\}+\{00101\}$ & $\mathrm{red} / \mathrm{red}$ & 0.227 & 0.017 & 13.654 & 0.262 & 0.211 & 1.242 \\
\hline 8 & $\{00100\}+\{10000,00001,00101,00111\}+\{10101\}$ & $\mathrm{red} / \mathrm{red}$ & 0.269 & 0.033 & 8.193 & 0.579 & 0.101 & 5.733 \\
\hline 7 & $\{00001\}+\{10000,00100,00101,00111\}+\{10101\}$ & $\mathrm{red} / \mathrm{red}$ & 0.269 & 0.033 & 8.193 & 0.579 & 0.101 & 5.733 \\
\hline 6 & $\{00001\}+\{10000,00010,00011,00111\}+\{10010\}$ & $\mathrm{red} / \mathrm{red}$ & 0.275 & 0.004 & 67.167 & 0.493 & 0.755 & 0.653 \\
\hline 5 & $\{00001\}+\{10000,00011,10011,00111\}+\{10010\}$ & $\mathrm{red} / \mathrm{red}$ & 0.275 & 0.004 & 67.167 & 0.493 & 0.755 & 0.653 \\
\hline 4 & $\{00110\}+\{10000,00100,10101,00111\}+\{00101\}$ & $\mathrm{red} / \mathrm{red}$ & 0.306 & 0.009 & 32.813 & 0.413 & 0.610 & 0.677 \\
\hline 3 & $\{00110\}+\{10000,10010,10110,00111\}+\{10111\}$ & red/blue & 0.344 & 0.024 & 14.403 & 0.186 & 0.035 & 5.345 \\
\hline \multirow[t]{2}{*}{2} & $\{00110\}+\{10000,00010,10010,00111\}+\{00011\}$ & red/blue & 0.354 & 0.024 & 14.760 & 0.175 & 0.030 & 5.853 \\
\hline & $\{00001\}+\{10000,10101,10011,00111\}+\{10111\}$ & $\mathrm{red} / \mathrm{blue}$ & 0.408 & 0.028 & 14.815 & 0.108 & 0.013 & 8.308 \\
\hline \multirow[t]{2}{*}{1} & $\{00100\}+\{10000,00010,00001,00111\}+\{00011\}$ & blue/red & 0.421 & 0.008 & 53.243 & 0.026 & 0.526 & 0.049 \\
\hline & $\{00110\}+\{10000,10110,10101,00111\}+\{10111\}$ & red/blue & 0.486 & 0.034 & 14.403 & 0.186 & 0.035 & 5.345 \\
\hline
\end{tabular}


Table S5: Parallel analysis GouldCFU $* 1 * * * \rightarrow$ Gould $* 1 * * *$, non-critical red/red-case omitted.

\begin{tabular}{|c|c|c|c|c|c|c|c|c|}
\hline No. & bipyramid & type & $e_{o}$ & $e_{p}$ & $e_{o} / e_{p}$ & $p_{o}$ & $p_{p}$ & $p_{o} / p_{p}$ \\
\hline 23 & $\{01100\}+\{11000,01110,01101,11110\}+\{11010\}$ & $\mathrm{red} / \mathrm{red}$ & 0.001 & 0.018 & 0.054 & 0.998 & 0.292 & 3.418 \\
\hline 22 & $\{01100\}+\{11000,01010,01001,01110\}+\{11010\}$ & $\mathrm{red} / \mathrm{red}$ & 0.001 & 0.018 & 0.054 & 0.998 & 0.292 & 3.418 \\
\hline 21 & $\{01100\}+\{11000,01001,01110,01101\}+\{11010\}$ & $\mathrm{red} / \mathrm{red}$ & 0.001 & 0.018 & 0.054 & 0.998 & 0.292 & 3.418 \\
\hline 20 & $\{11001\}+\{11000,01001,11101,11011\}+\{11111\}$ & red/blue & 0.002 & 0.059 & 0.033 & 0.962 & 0.001 & 1286.096 \\
\hline 19 & $\{11110\}+\{11010,01110,01101,11111\}+\{01111\}$ & $\mathrm{red} / \mathrm{red}$ & 0.005 & 0.009 & 0.488 & 0.945 & 0.576 & 1.641 \\
\hline 18 & $\{11100\}+\{11000,01100,11110,11101\}+\{01101\}$ & $\mathrm{red} / \mathrm{red}$ & 0.005 & 0.023 & 0.218 & 0.952 & 0.193 & 4.933 \\
\hline 17 & $\{11000\}+\{11010,01110,01101,11110\}+\{11111\}$ & $\mathrm{red} / \mathrm{red}$ & 0.010 & 0.026 & 0.369 & 0.981 & 0.106 & 9.255 \\
\hline 16 & $\{01110\}+\{11000,11010,01101,11110\}+\{11111\}$ & $\mathrm{red} / \mathrm{red}$ & 0.010 & 0.026 & 0.369 & 0.981 & 0.106 & 9.255 \\
\hline 15 & $\{01100\}+\{11000,01101,11110,11101\}+\{11111\}$ & $\mathrm{red} / \mathrm{red}$ & 0.013 & 0.014 & 0.905 & 0.866 & 0.346 & 2.503 \\
\hline 14 & $\{11000\}+\{01001,11010,01110,01101\}+\{01111\}$ & $\mathrm{red} / \mathrm{red}$ & 0.013 & 0.020 & 0.656 & 0.974 & 0.160 & 6.087 \\
\hline 13 & $\{11000\}+\{01001,11010,01101,11111\}+\{01111\}$ & $\mathrm{red} / \mathrm{red}$ & 0.013 & 0.020 & 0.656 & 0.974 & 0.160 & 6.087 \\
\hline 12 & $\{01000\}+\{11000,01100,01010,01001\}+\{01110\}$ & $\mathrm{red} / \mathrm{red}$ & 0.024 & 0.015 & 1.584 & 0.783 & 0.433 & 1.808 \\
\hline 11 & $\{11010\}+\{11000,01001,01101,11111\}+\{11101\}$ & $\mathrm{red} / \mathrm{red}$ & 0.059 & 0.017 & 3.428 & 0.905 & 0.318 & 2.846 \\
\hline 10 & $\{11010\}+\{11000,01001,11011,11111\}+\{11101\}$ & $\mathrm{red} / \mathrm{red}$ & 0.059 & 0.017 & 3.428 & 0.905 & 0.318 & 2.846 \\
\hline 9 & $\{11010\}+\{11000,01101,11110,11111\}+\{11101\}$ & $\mathrm{red} / \mathrm{red}$ & 0.059 & 0.017 & 3.428 & 0.905 & 0.318 & 2.846 \\
\hline 8 & $\{01010\}+\{01001,11010,01110,01011\}+\{01111\}$ & $\mathrm{red} / \mathrm{red}$ & 0.067 & 0.018 & 3.722 & 0.323 & 0.186 & 1.737 \\
\hline 7 & $\{01110\}+\{01001,11010,01101,01111\}+\{11111\}$ & $\mathrm{red} / \mathrm{red}$ & 0.075 & 0.022 & 3.483 & 0.841 & 0.136 & 6.184 \\
\hline 6 & $\{01001\}+\{11010,01110,01101,01111\}+\{11111\}$ & $\mathrm{red} / \mathrm{red}$ & 0.075 & 0.022 & 3.483 & 0.841 & 0.136 & 6.184 \\
\hline 5 & $\{11011\}+\{01001,11010,01011,11111\}+\{01111\}$ & $\mathrm{red} / \mathrm{red}$ & 0.081 & 0.000 & 1235.241 & 0.126 & 0.996 & 0.127 \\
\hline 4 & $\{11000\}+\{01010,01001,11010,01110\}+\{01011\}$ & red/blue & 0.170 & 0.030 & 5.666 & 0.718 & 0.026 & 27.722 \\
\hline 3 & $\{11000\}+\{01001,11010,11011,11111\}+\{01011\}$ & $\mathrm{red} / \mathrm{blue}$ & 0.170 & 0.030 & 5.666 & 0.718 & 0.026 & 27.722 \\
\hline 2 & $\{01101\}+\{01001,11010,01110,01111\}+\{01011\}$ & $\mathrm{red} / \mathrm{red}$ & 0.192 & 0.013 & 15.097 & 0.533 & 0.362 & 1.472 \\
\hline 1 & $\{01101\}+\{01001,11010,01111,11111\}+\{01011\}$ & $\mathrm{red} / \mathrm{red}$ & 0.192 & 0.013 & 15.097 & 0.533 & 0.362 & 1.472 \\
\hline
\end{tabular}


Table S6: Significant 4-dimensional interactions, which cannot be seen in lower dimensions, cf. (Fig. S20). The value $p \uparrow$ refers to the $p$-value of the 4-dimensional bipyramid in question whereas $p \downarrow$ is the $p$-value of its ridge intersected with the $\cap$-face, cf. (Fig. S20c) for the Gould data.

\begin{tabular}{lcccc}
\hline Data & significant bipyramid & $\cap$-face & $p \uparrow$ & $p \downarrow$ \\
\hline Eble & - & - & - & - \\
\hline Gould & $\{00010\}+\{00000,10010,00011,11011\}+\{10001\}$ & $* * 01 *$ & 0.041 & 0.270 \\
$* * 0 * *$ & $\{10010\}+\{00000,11000,10001,11011\}+\{01001\}$ & $1 * 0 * *$ & 0.041 & 0.076 \\
& & $* * 00 *$ & 0.041 & 0.063 \\
& & & & \\
& & & & \\
Khan & $\{00010\}+\{00000,01001,00101,00011\}+\{00001\}$ & $0 * * * 1$ & 0.009 & 0.052 \\
$0 * * * *$ & & & & \\
\hline
\end{tabular}


Table S7: Bacterial species considered in the two microbiome data sets.

\begin{tabular}{lrr}
\hline & Gould data set & Eble data set \\
\hline Species 1 & L. plantarum & L. plantarum \\
Species 2 & L. brevis & L. brevis \\
Species 3 & A. pasteurianus & A. cerevisiae \\
Species 4 & A. tropicalis & A. malorum \\
Species 5 & A. orientalis & A. orientalis \\
\hline
\end{tabular}


Table S8: Parallel analysis Eble $0 * * * * \rightarrow 1 * * * *$, non-critical red/red-case omitted.

\begin{tabular}{|c|c|c|c|c|c|c|c|c|}
\hline No. & bipyramid $_{s}$ & type & $e_{o}$ & $e_{p}$ & $e_{o} / e_{p}$ & $p_{o}$ & $p_{p}$ & $p_{o} / p_{p}$ \\
\hline 23 & $\{00001\}+\{00000,01001,01011,00111\}+\{01111\}$ & $\mathrm{red} / \mathrm{red}$ & 0.001 & 0.012 & 0.066 & 0.953 & 0.390 & 2.444 \\
\hline 22 & $\{00001\}+\{00000,01001,01101,00111\}+\{01111\}$ & $\mathrm{red} / \mathrm{red}$ & 0.001 & 0.012 & 0.066 & 0.953 & 0.390 & 2.444 \\
\hline 21 & $\{01110\}+\{00000,00110,01011,01111\}+\{00111\}$ & red/blue & 0.001 & 0.025 & 0.041 & 0.923 & 0.038 & 24.226 \\
\hline 20 & $\{01110\}+\{00000,01100,00110,01111\}+\{00111\}$ & red/blue & 0.001 & 0.035 & 0.041 & 0.923 & 0.038 & 24.226 \\
\hline 19 & $\{00110\}+\{00000,01100,00111,01111\}+\{01101\}$ & $\mathrm{red} / \mathrm{red}$ & 0.002 & 0.012 & 0.201 & 0.827 & 0.303 & 2.729 \\
\hline 18 & $\{00110\}+\{00000,01100,00101,00111\}+\{01101\}$ & $\mathrm{red} / \mathrm{red}$ & 0.003 & 0.014 & 0.201 & 0.827 & 0.303 & 2.729 \\
\hline 17 & $\{01110\}+\{00000,01000,01100,01111\}+\{01101\}$ & $\mathrm{red} / \mathrm{red}$ & 0.003 & 0.013 & 0.264 & 0.742 & 0.251 & 2.956 \\
\hline 16 & $\{00110\}+\{00000,00010,01011,00111\}+\{00011\}$ & $\mathrm{red} / \mathrm{red}$ & 0.004 & 0.003 & 1.568 & 0.755 & 0.843 & 0.896 \\
\hline 15 & $\{00010\}+\{00000,01010,00110,01011\}+\{01110\}$ & $\mathrm{red} / \mathrm{red}$ & 0.007 & 0.010 & 0.748 & 0.606 & 0.488 & 1.242 \\
\hline 14 & $\{01010\}+\{00000,00010,00110,01011\}+\{00111\}$ & $\mathrm{red} / \mathrm{red}$ & 0.008 & 0.005 & 1.583 & 0.443 & 0.639 & 0.693 \\
\hline 13 & $\{01010\}+\{00000,00110,01110,01011\}+\{01111\}$ & $\mathrm{red} / \mathrm{red}$ & 0.009 & 0.024 & 0.359 & 0.475 & 0.062 & 7.686 \\
\hline 12 & $\{01010\}+\{00000,01000,01110,01011\}+\{01111\}$ & $\mathrm{red} / \mathrm{red}$ & 0.009 & 0.024 & 0.359 & 0.475 & 0.062 & 7.686 \\
\hline 11 & $\{00100\}+\{00000,01100,00110,00101\}+\{00111\}$ & $\mathrm{red} / \mathrm{red}$ & 0.009 & 0.018 & 0.498 & 0.533 & 0.269 & 1.981 \\
\hline 10 & $\{01001\}+\{00000,00001,01101,00111\}+\{00101\}$ & $\mathrm{red} / \mathrm{red}$ & 0.014 & 0.014 & 1.018 & 0.288 & 0.313 & 0.920 \\
\hline \multirow[t]{2}{*}{9} & $\{00101\}+\{00000,01100,01101,00111\}+\{01111\}$ & $\mathrm{red} / \mathrm{red}$ & 0.015 & 0.026 & 0.584 & 0.228 & 0.062 & 3.695 \\
\hline & $\{00101\}+\{00000,01100,00110,00111\}+\{01111\}$ & $\mathrm{red} / \mathrm{blue}$ & 0.018 & 0.040 & 0.446 & 0.321 & 0.035 & 9.119 \\
\hline 8 & $\{01101\}+\{00000,01001,00111,01111\}+\{01011\}$ & $\mathrm{red} / \mathrm{red}$ & 0.019 & 0.003 & 6.623 & 0.068 & 0.800 & 0.085 \\
\hline 7 & $\{01101\}+\{00000,01000,01001,01111\}+\{01011\}$ & $\mathrm{red} / \mathrm{red}$ & 0.019 & 0.003 & 6.623 & 0.068 & 0.800 & 0.085 \\
\hline 6 & $\{01001\}+\{00000,00001,01011,00111\}+\{00011\}$ & $\mathrm{red} / \mathrm{red}$ & 0.019 & 0.005 & 3.571 & 0.153 & 0.689 & 0.222 \\
\hline 5 & $\{01000\}+\{00000,01010,01110,01011\}+\{00110\}$ & $\mathrm{red} / \mathrm{red}$ & 0.020 & 0.011 & 1.750 & 0.169 & 0.443 & 0.381 \\
\hline 4 & $\{01000\}+\{00000,01100,01110,01111\}+\{00110\}$ & $\mathrm{red} / \mathrm{red}$ & 0.020 & 0.011 & 1.750 & 0.169 & 0.443 & 0.381 \\
\hline 3 & $\{01000\}+\{00000,01001,01011,01111\}+\{00111\}$ & $\mathrm{red} / \mathrm{red}$ & 0.021 & 0.013 & 1.535 & 0.140 & 0.339 & 0.413 \\
\hline 2 & $\{01100\}+\{00000,01000,01101,01111\}+\{01001\}$ & blue/blue & 0.045 & 0.037 & 1.215 & 0.000 & 0.003 & 0.176 \\
\hline \multirow[t]{8}{*}{1} & $\{01001\}+\{00000,01000,01011,01111\}+\{01110\}$ & blue/red & 0.048 & 0.024 & 1.993 & 0.000 & 0.056 & 0.002 \\
\hline & $\{01100\}+\{00000,01000,01110,01111\}+\{01011\}$ & blue/blue & 0.064 & 0.034 & 1.855 & 0.000 & 0.005 & 0.000 \\
\hline & $\{00010\}+\{00000,00011,01011,00111\}+\{00001\}$ & blue/blue & 0.065 & 0.043 & 1.518 & 0.000 & 0.001 & 0.001 \\
\hline & $\{01100\}+\{00000,01101,00111,01111\}+\{01001\}$ & blue/red & 0.066 & 0.024 & 2.775 & 0.000 & 0.105 & 0.000 \\
\hline & $\{00001\}+\{00000,00101,01101,00111\}+\{01100\}$ & blue/blue & 0.066 & 0.033 & 1.989 & 0.000 & 0.009 & 0.000 \\
\hline & $\{01001\}+\{00000,01011,00111,01111\}+\{00110\}$ & blue/blue & 0.068 & 0.036 & 1.917 & 0.000 & 0.007 & 0.000 \\
\hline & $\{01100\}+\{00000,00110,01110,01111\}+\{01011\}$ & blue/blue & 0.083 & 0.045 & 1.829 & 0.000 & 0.002 & 0.000 \\
\hline & $\{01100\}+\{00000,00110,00111,01111\}+\{01011\}$ & blue/red & 0.084 & 0.021 & 4.035 & 0.000 & 0.210 & 0.000 \\
\hline
\end{tabular}


Table S9: Regressions over $\{0001\}+\{0000,1001,1011,0111\}+\{1111\}$ for normalized lifespan data for Eble $0 * * * *$ and Eble $1 * * * *$.

\begin{tabular}{lrrrr}
\hline & Coefficient & Std. error & $t$-statistic & $p$-value \\
\hline$\beta_{0}$ & 0 & 0 & nan & nan \\
$x_{1}$ & -0.0270 & 0.009 & -2.987 & 0.003 \\
$x_{2}$ & -0.0149 & 0.012 & -1.246 & 0.213 \\
$x_{3}$ & -0.0156 & 0.012 & -1.306 & 0.192 \\
$x_{4}$ & 0.2039 & 0.008 & 26.022 & 0.000 \\
\hline$\beta_{0}$ & 0.2320 & 0.005 & 44.642 & 0.000 \\
$x_{1}$ & 0.0310 & 0.005 & 5.957 & 0.000 \\
$x_{2}$ & 0.0610 & 0.007 & 8.874 & 0.000 \\
$x_{3}$ & -0.0185 & 0.007 & -2.692 & 0.007 \\
$x_{4}$ & -0.0861 & 0.007 & -12.518 & 0.000 \\
\hline
\end{tabular}

